\title{
Aspects of multivariable operator theory on weighted symmetric Fock spaces
}

\author{
H. Turgay Kaptanoğlu \\ Department of Mathematics, Bilkent University \\ 06800 Ankara, Turkey \\ kaptan@fen.bilkent.edu.tr \\ http://www.fen.bilkent.edu.tr/ kaptan/ \\ Received 28 February 2013 \\ Revised 19 June 2013 \\ Accepted 2 July 2013 \\ Published 26 September 2013
}

\begin{abstract}
We obtain all Dirichlet spaces $\mathcal{F}_{q}, q \in \mathbb{R}$, of holomorphic functions on the unit ball of $\mathbb{C}^{N}$ as weighted symmetric Fock spaces over $\mathbb{C}^{N}$. We develop the basics of operator theory on these spaces related to shift operators. We do a complete analysis of the effect of $q \in \mathbb{R}$ in the topics we touch upon. Our approach is concrete and explicit. We use more function theory and reduce many proofs to checking results on diagonal operators on the $\mathcal{F}_{q}$. We pick out the analytic Hilbert modules from among the $\mathcal{F}_{q}$. We obtain von Neumann inequalities for row contractions on a Hilbert space with respect to each $\mathcal{F}_{q}$. We determine the commutants and investigate the almost normality of the shift operators. We prove that the $C^{*}$-algebras generated by the shift operators on the $\mathcal{F}_{q}$ fit in exact sequences that are in the same Ext class. We identify the groups $K_{0}$ and $K_{1}$ of the Toeplitz algebras on the $\mathcal{F}_{q}$ arising in $K$-theory. Radial differential operators are prominent throughout. Some of our results, especially those pertaining to lower negative values of $q$, are new even for $N=1$. Many of our results are valid in the more general weighted symmetric Fock spaces $\mathcal{F}_{b}$ that depend on a weight sequence $b$.
\end{abstract}

Keywords: Fock; Dirichlet; Drury-Arveson; Hardy; Bergman; reproducing kernel Hilbert space; radial differential operator; multiplier; virtual point; analytic Hilbert module; shift; row contraction; von Neumann inequality; spectrum; commutant; Fredholm; hyponormal; subnormal; Toeplitz; $C^{*}$-algebra; short exact sequence; extension; Busby invariant; $K$-groups.

Mathematics Subject Classification 2010: 47A13, 47B32, 19K33, 32A36, 32A37, 46E20, 46E22, 46L08, 47A20, 47A30, 47A53, 47B35, 47B37, 47B38, 47C15

\section{Introduction}

The purpose of this paper is to investigate certain aspects of multivariable operator theory on a family of Hilbert spaces that are weighted symmetric Fock spaces. The spaces in this family are realized as Hilbert spaces of holomorphic functions defined on the unit ball $\mathbb{B}$ of $\mathbb{C}^{N}$ and include the standard weighted Bergman spaces, the 
Hardy space, the Drury-Arveson space, and the Dirichlet space as special cases. We name them the Dirichlet spaces $\mathcal{F}_{q}$ indexed by $q \in \mathbb{R}$. We focus on identifying the similarities and differences among various Hilbert spaces from the operatortheoretic point of view as $q$ varies. This also highlights why and how the DruryArveson space is so special among the Dirichlet spaces. We concentrate mostly on $N>1$, but some of our results are new even for $N=1$.

Our starting point is noticing that the Drury-Arveson space $\mathcal{A}$ defined as a symmetric Fock space is one of the Dirichlet spaces which is the Hilbert subfamily of the Besov spaces on $\mathbb{B}$. We want to investigate how much of the operator theory developed for $\mathcal{A}$ can be extended to the remaining Hilbert spaces and how. We do our work by obtaining all the $\mathcal{F}_{q}$ as weighted symmetric Fock spaces. It turns out that we can be even more general than the Dirichlet family by starting with a quite general weight sequence.

Our work has its beginnings in [9], which deals solely with the Drury-Arveson space $\mathcal{A}$ which is our space $\mathcal{F}_{-N}$. Several other sources deal with a family of spaces that are reproducing kernel Hilbert spaces on $\mathbb{B}$ whose reproducing kernels are powers of the Bergman kernel. These correspond to $q>-(1+N)$ in our family of Dirichlet spaces. However, for $q \leq-(1+N)$ as well, the Dirichlet spaces $\mathcal{F}_{q}$ are reproducing kernel Hilbert spaces on $\mathbb{B}$ whose reproducing kernels are hypergeometric functions that converge on $\mathbb{B}$, and this is sufficient for many applications. This subfamily has largely been ignored due to a lack of information about the reproducing kernels of the spaces although the Dirichlet space itself is $\mathcal{F}_{-(1+N)}$.

Working with holomorphic function spaces on $\mathbb{B}$ that are also reproducing kernel Hilbert spaces allows us to use more function theory than usual. We obtain several of our results on the Taylor (or homogeneous) series expansions of the functions in the spaces, because many interesting operators turn out to be diagonal operators. This actually simplifies our work and helps us to obtain explicit formulas. Thus many of our proofs are more concrete and thus more understandable. Rather than trying to obtain the most general results, we completely analyze the effect of $q \in \mathbb{R}$. In several places, we can easily be more general and speak of the weighted symmetric Fock spaces $\mathcal{F}_{b}$ depending on a weight sequence $b=\left\{b_{k}\right\}$.

Here is a synopsis of the paper. In Sec. 3, we obtain the generalized Dirichlet spaces as weighted symmetric Fock spaces. In Sec. 4, we consider those Dirichlet spaces that are Hilbert Besov spaces of holomorphic functions on $\mathbb{B}$. Here radial differential operators are the central idea. In Sec. 5, we identify those Dirichlet spaces that are analytic Hilbert modules. In Sec. 6, we obtain many formulas for the shift operators acting on the Dirichlet spaces that we need later. We notice that they are very similar to radial differential operators. In Sec. 7, we prove that there is a von Neumann inequality for row contractions on any Hilbert space with respect to any Dirichlet space using a dilation theorem. In Sec. 8, we investigate the spectra of shift operators in some detail when $N=1$, because then they turn out to be weighted shifts. It is here that we also determine the commutants of the shift operators in all dimensions. In Sec. 9, we investigate how much of various 
kinds of almost normality is present in the shift operators on the Dirichlet spaces. In Sec. 10, we show that the $C^{*}$-algebra generated by the shift operators on each Dirichlet space fits in a short exact sequence. This in turn yields information about the Fredholm properties of the elements of the $C^{*}$-algebra and other related objects. In Sec. 11, we show that all the short exact sequences obtained are in the same Ext class as well as computing the $K$-theory groups $K_{0}$ and $K_{1}$ of these $C^{*}$-algebras.

\section{Notation}

We start by introducing the basic notation. The usual Hermitian inner product in $\mathbb{C}^{N}$ is $\langle z, w\rangle=z_{1} \bar{w}_{1}+\cdots+z_{N} \bar{w}_{N}$ with the associated norm $|z|=\sqrt{\langle z, z\rangle}$, where the overline represents the complex conjugate. This gives rise to the orthonormal basis $\left\{e_{1}, \ldots, e_{N}\right\}$ of $\mathbb{C}^{N}$, where $e_{j}=(0, \ldots, 0,1,0, \ldots, 0)$ with 1 in the $j$ th position. We use multi-index notation in which $\alpha=\left(\alpha_{1}, \ldots, \alpha_{N}\right) \in \mathbb{N}^{N}$ is an $N$-tuple of nonnegative integers, $|\alpha|=\alpha_{1}+\cdots+\alpha_{N}, \alpha !=\alpha_{1} ! \cdots \alpha_{N} !, z^{\alpha}=z_{1}^{\alpha_{1}} \cdots z_{N}^{\alpha_{N}}$, and $0^{0}=1$, where $z=\left(z_{1}, \ldots, z_{N}\right) \in \mathbb{C}^{N}$.

We denote the unit ball of $\mathbb{C}^{N}$ with respect to the usual norm by $\mathbb{B}$ and its boundary the unit sphere by $\partial \mathbb{B}$. When $N=1$, the unit ball is the unit disc $\mathbb{D}$. We denote the space of holomorphic functions on $\mathbb{B}$ by $H(\mathbb{B})$ and the algebra of bounded holomorphic functions on $\mathbb{B}$ by $H^{\infty}$. The ball algebra $A(\mathbb{B})$ consists of holomorphic functions on $\mathbb{B}$ that extend continuously to its closure $\overline{\mathbb{B}}$. We denote the space of continuous functions on a compact set $K$ by $C(K)$. The last three spaces are adorned with the supremum norm.

Let $\nu$ be the volume measure on $\mathbb{C}^{N}$ normalized as $\nu(\mathbb{B})=1$. For $q \in \mathbb{R}$, we define on $\mathbb{B}$ also the measures

$$
d \nu_{q}(z)=C_{q}\left(1-|z|^{2}\right)^{q} d \nu(z)
$$

These measures are finite only for $q>-1$ and then we choose $C_{q}$ so that $\nu_{q}(\mathbb{B})=1$. For $q \leq-1$, we set $C_{q}=1$. For $0<p<\infty$, we denote the Lebesgue classes with respect to $\nu_{q}$ by $\mathcal{L}_{q}^{p}$ and the sequence spaces whose $p$ th powers are summable by $\ell^{p}$.

The Pochhammer symbol $(x)_{y}$ for $x, y \in \mathbb{C}$ is defined by

$$
(x)_{y}=\frac{\Gamma(x+y)}{\Gamma(x)}
$$

when $x$ and $x+y$ are off the pole set $-\mathbb{N}$ of the gamma function $\Gamma$. This is a shifted factorial since $(x)_{k}=x(x+1) \cdots(x+k-1)$ for positive integer $k$. In particular, $(1)_{k}=k$ ! and $(x)_{0}=1$. The Stirling formula gives

$$
\frac{\Gamma(c+x)}{\Gamma(c+y)} \sim c^{x-y}, \quad \frac{(x)_{c}}{(y)_{c}} \sim c^{x-y} \quad \text { and } \quad \frac{(c)_{x}}{(c)_{y}} \sim c^{x-y} \quad(\operatorname{Re} c \rightarrow \infty),
$$

where $A \sim B$ means that $|A / B|$ is bounded above and below by two positive constants. Such constants that are independent of the parameters in the equation are all denoted by the generic unadorned upper case $C$. 
The classical hypergeometric function is

$$
{ }_{2} F_{1}(a, b ; c ; z)=\sum_{k=0}^{\infty} \frac{(a)_{k}(b)_{k}}{(c)_{k}(1)_{k}} z^{k} \quad(a, b \in \mathbb{R}, c>0, z \in \mathbb{D}) .
$$

The series converges absolutely and uniformly for $z$ in compact subsets of $\mathbb{B}$; therefore ${ }_{2} F_{1} \in H(\mathbb{B})$.

Consider the space $\mathcal{P}_{k}$ of all holomorphic polynomials that are homogeneous of degree $k$. Elements of $\mathcal{P}_{k}$ have the form $\sum_{|\alpha|=k} c_{\alpha} z^{\alpha}$. The dimension of $\mathcal{P}_{k}$ is

$$
\delta_{k}=\frac{(N)_{k}}{k !} \sim k^{N-1} \quad(k \rightarrow \infty) .
$$

We let $P_{k}$ denote the orthogonal projection from a larger Hilbert space onto $\mathcal{P}_{k}$.

The algebra of all bounded operators on a complex separable Hilbert space $H$ is denoted $\mathcal{B}(H)$ and of all compact operators $\mathcal{K}(H)$. The spectrum and the point spectrum (the set of eigenvalues) of an operator $T \in \mathcal{B}(H)$ are denoted $\sigma(T)$ and $\sigma_{p}(T)$, respectively, as usual. If the inner product of $H$ is $[\cdot, \cdot], T$ is called positive and we write $T \geq 0$ if $[T v, v] \geq 0$ for all $v \in H$. For $a, b \in H, a \otimes b$ denotes the rank-1 operator defined by $(a \otimes b)(v)=[v, b] a$ for $v \in H$.

The commutant $\{\cdots\}^{\prime}$ of a set $\{\cdots\}$ of bounded operators on $H$ is the set of all bounded operators on $H$ that commute with each operator in $\{\cdots\}$, and is an algebra containing the identity operator $I$. The double commutant $\{\cdots\}^{\prime \prime}$ is the commutant of the commutant. A net of bounded operators $\left\{T_{\ell}\right\}$ on $H$ converges to $T \in \mathcal{B}(H)$ in the strong operator topology (sot) if $T_{\ell} v \rightarrow T v$ for all $v \in H$; the convergence is in the weak operator topology $(w o t)$ if $\left[T_{\ell} v, u\right] \rightarrow[T v, u]$ for all $v, u \in H$.

The singular values of $T$ are the eigenvalues of the positive operator $\left(T^{*} T\right)^{1 / 2}$, where $T^{*}$ is the adjoint of $T$. For $0<p<\infty, T$ belongs to the Schatten class $\mathcal{C}^{p}(H)$ if its sequence of singular values belongs to $\ell^{p}$. Of course, $\mathcal{C}^{p}(H) \subset \mathcal{K}(H)$, and $\mathcal{K}(H)$ and $\mathcal{C}^{p}(H)$ are ideals of $\mathcal{B}(H)$. The Banach space $\mathcal{B}(H)$ can be realized as the dual of $\mathcal{C}^{1}(H)$, and the topology on $\mathcal{B}(H)$ induced by this duality is called the weak $^{*}$ (ultraweak) topology of $\mathcal{B}(H)$. The quotient $\mathcal{Q}(H):=\mathcal{B}(H) / \mathcal{K}(H)$ is called the Calkin algebra. The essential spectrum $\sigma_{e}(T)$ and the essential norm $\|T\|_{e}$ of $T$ are the spectrum and the norm of the coset $[T]:=T+\mathcal{K}(H)$ of $T$ in $\mathcal{Q}(H)$, respectively.

As usual, the abbreviations dim, ker, and im denote the dimension, kernel, and image (range), respectively. We use terms like positive and increasing loosely to mean nonnegative and nondecreasing. The right-hand side of $:=$ defines its lefthand side.

\section{Weighted Symmetric Fock Spaces}

The symmetric tensor product allows one to carry out multivariable operator theory by pretending working with a single variable. We modify and generalize this construction by adding appropriate weights. 
Following the notation of [9], let $E=\mathbb{C}^{N}$ and denote by $E^{k}$ the subspace of the tensor product $E^{\otimes k}$ of $k$ copies of $E$ which is fixed elementwise under the action of the permutation group on $k$ objects. So $E^{k}$ is spanned by the $\operatorname{set}\left\{z^{\otimes k}: z \in E\right\}$. For completeness we set $E^{0}=\mathbb{C}$ and $z^{\otimes 0}=1$. The usual inner product and norm on $E^{k}$ are the restrictions of those on $E^{\otimes k}$ and are given on elementary tensors by $\left\langle z^{\otimes k}, w^{\otimes k}\right\rangle_{k}=\langle z, w\rangle^{k}$ and $\left|z^{\otimes k}\right|_{k}=|z|^{k}$, but we need to introduce weights to accommodate a whole family of spaces.

For this, we start with a weight sequence $b=\left\{b_{k}: k=0,1,2, \ldots\right\}$ of strictly positive real numbers with $b_{0}=1$ and satisfying

$$
Z_{b}:=\frac{1}{\limsup _{k \rightarrow \infty} b_{k}^{1 / k}} \geq 1 .
$$

Note that (3) implies nothing about the boundedness, summability, or monotoneness of $b$. The coefficient sequence of any power series in one variable whose disc of convergence includes $\mathbb{D}$ is acceptable for $b$. This kind of $b$ is more general than what is called regular in [26, p. 231] by [40, Example 3.35]. In most practical cases, $b_{k}$ acts like a fixed power of $k$.

We endow $E^{k}$ with the weighted inner product defined on elementary tensors by

$$
\left[z^{\otimes k}, w^{\otimes k}\right]_{k}:=b_{k}\left\langle z^{\otimes k}, w^{\otimes k}\right\rangle_{k}=b_{k}\langle z, w\rangle^{k}
$$

and extended linearly to all of $E^{k}$. Thus $E^{\otimes k}$ and $E^{k}$ are Hilbert spaces of dimensions $N^{k}$ and $N$, respectively. Let us denote the corresponding norm on $E^{k}$ by $\|\cdot\|_{k} ;$ so

$$
\left\|z^{\otimes k}\right\|_{k}^{2}:=b_{k}\left\langle z^{\otimes k}, z^{\otimes k}\right\rangle_{k}=b_{k}|z|^{2 k} .
$$

Every holomorphic homogeneous polynomial $f_{k}: \mathbb{C}^{N} \rightarrow \mathbb{C}$ of degree $k$ gives rise to a unique linear functional $\tilde{f}_{k}: E^{k} \rightarrow \mathbb{C}$ by $\tilde{f}_{k}\left(z^{\otimes k}\right)=f_{k}(z)$. There exists a unique $\zeta_{k} \in E^{k}$ such that $\tilde{f}_{k}\left(z^{\otimes k}\right)=\left[z^{\otimes k}, \zeta_{k}\right]_{k}$ for all $z \in \mathbb{C}^{N}$ by the Riesz representation theorem. In particular, $\zeta_{0}=\bar{f}_{0}$. For the monomial $f_{k}(z)=z_{1}^{k}$, we compute easily that $\zeta_{k}=\left(b_{k}^{-1 / k}, 0, \ldots, 0\right)^{\otimes k}$. If $f$ is a polynomial of degree $n$ with homogeneous expansion $f=f_{0}+f_{1}+\cdots+f_{n}$, then

$$
\begin{aligned}
f(z) & =\left[1, \zeta_{0}\right]_{0}+\left[z, \zeta_{1}\right]_{1}+\left[z \otimes z, \zeta_{2}\right]_{2}+\cdots+\left[z^{\otimes n}, \zeta_{n}\right]_{n} \\
& =\bar{\zeta}_{0}+b_{1}\left\langle z, \zeta_{1}\right\rangle+b_{2}\left\langle z \otimes z, \zeta_{2}\right\rangle_{2}+\cdots+b_{n}\left\langle z^{\otimes n}, \zeta_{n}\right\rangle_{n} \quad\left(z \in \mathbb{C}^{N}\right) .
\end{aligned}
$$

This representation induces on polynomials an inner product $[\cdot, \cdot]_{b}$ whose associated norm $\|\cdot\|_{b}$ is given by

$$
\|f\|_{b}^{2}:=\left\|\zeta_{0}\right\|_{0}^{2}+\left\|\zeta_{1}\right\|_{1}^{2}+\cdots+\left\|\zeta_{n}\right\|_{n}^{2}=\left|\zeta_{0}\right|^{2}+b_{1}\left|\zeta_{1}\right|_{1}^{2}+\cdots+b_{n}\left|\zeta_{n}\right|_{n}^{2} .
$$

By construction, $\left\|\zeta_{0}\right\|=\left|f_{0}\right|$ and $\left[f_{k}, f_{l}\right]_{b}=0$ if $k \neq l$.

Definition 3.1. We denote by $\mathcal{F}_{b}$ the Hilbert space obtained by completing the polynomials in the norm $\|\cdot\|_{b}$. 
So $\mathbb{C} \subset \mathcal{F}_{b}$ and $\mathcal{F}_{b}$ is separable. Next we identify $\mathcal{F}_{b}$ with the weighted symmetric Fock space

$$
\mathcal{E}_{b}(E)=E^{0} \oplus E^{1} \oplus E^{2} \oplus \cdots
$$

(called also a bosonic Fock space by physicists) over $E$, where each $E^{k}$ is taken with the norm $\|\cdot\|_{k}$. A function $f \in \mathcal{F}_{b}$ has the Taylor (homogeneous) expansion

$$
f(z)=\sum_{k=0}^{\infty}\left[z^{\otimes k}, \zeta_{k}\right]_{k}=\sum_{k=0}^{\infty} b_{k}\left\langle z^{\otimes k}, \zeta_{k}\right\rangle_{k}=\sum_{k=0}^{\infty} f_{k}(z),
$$

where $G f:=\left(\zeta_{0}, \zeta_{1}, \zeta_{2} \ldots\right) \in \mathcal{E}_{b}(E)$ is the sequence of Taylor coefficients. Starting with $\left(\zeta_{0}, \zeta_{1}, \zeta_{2} \ldots\right)$, an $f$ it defines belongs to $\mathcal{F}_{b}$ if and only if

$$
\|f\|_{b}^{2}:=\sum_{k=0}^{\infty}\left\|f_{k}\right\|_{k}^{2}=\sum_{k=0}^{\infty}\left\|\zeta_{k}\right\|_{k}^{2}=\sum_{k=0}^{\infty} b_{k}\left|\zeta_{k}\right|_{k}^{2}<\infty .
$$

The right-hand side of this equation also defines the corresponding norm $\|\cdot\|_{b}$ on $\mathcal{E}_{b}(E)$. Because the Taylor coefficients of $f$ appear in the second position in each term in (5), the correspondence $f \mapsto G f$ is conjugate linear and then we have $[f, g]_{b}=[G g, G f]_{b}$. With this identification, any homogeneous polynomial $f_{k}$ of degree $k$ can be considered as an element of $E^{k}$ since $G f_{k}$ has only one nonzero term which is in $E^{k}$. Hence $f_{k}(z)=z^{\alpha} \in E^{k}$ if $|\alpha|=k$.

Further, we define

$$
K_{b}(z, w):=\sum_{k=0}^{\infty} b_{k}\langle z, w\rangle^{k}
$$

where the series converges absolutely for $z, w \in \mathbb{B}$ and uniformly on compact subsets by (3). Then

$$
\begin{aligned}
|f(z)| & \leq \sum_{k=0}^{\infty} b_{k}\left|\left\langle z^{\otimes k}, \zeta_{k}\right\rangle_{k}\right| \leq \sum_{k=0}^{\infty} b_{k}\left|z^{\otimes k}\right|_{k}\left|\zeta_{k}\right|_{k}=\sum_{k=0}^{\infty} \sqrt{b_{k}}|z|^{k} \sqrt{b_{k}}\left|\zeta_{k}\right|_{k} \\
& \leq\left(\sum_{k=0}^{\infty} b_{k}|z|^{2 k}\right)^{1 / 2}\left(\sum_{k=0}^{\infty} b_{k}\left|\zeta_{k}\right|_{k}^{2}\right)^{1 / 2}=\sqrt{K_{b}(z, z)}\|f\|_{b}
\end{aligned}
$$

Observe that

$$
K_{b}(z, z) \geq 1 \quad(z \in \mathbb{B})
$$

since we have picked $b_{k}>0$ for all $k$ and $b_{0}=1$. Let us sum up.

Theorem 3.2. An element $f$ of the space $\mathcal{F}_{b}$ is a holomorphic function on $\mathbb{B}$. It has the Taylor expansion (5) on $\mathbb{B}$ which converges absolutely and uniformly on compact subsets of $\mathbb{B}$. The evaluation of $f$ at $z \in \mathbb{B}$ is a bounded linear functional on $\mathcal{F}_{b}$ of norm at most $\sqrt{K_{b}(z, z)}$. 
Noting that

$$
K_{b}(z, w)=\sum_{k=0}^{\infty} b_{k}\langle z, w\rangle^{k}=\sum_{k=0}^{\infty} b_{k}\left\langle z^{\otimes k}, w^{\otimes k}\right\rangle_{k}=\sum_{k=0}^{\infty}\left[z^{\otimes k}, w^{\otimes k}\right]_{k}
$$

and comparing with $(4)$, we see that $G K_{b}(\cdot, w)=\left(1, w, w \otimes w, w^{\otimes 3}, \ldots\right)$; that is, $\zeta_{k}=w^{\otimes k}$ for $k=0,1,2, \ldots$ for $K_{b}(\cdot, w)$. Since

$$
\left\|G K_{b}\right\|_{b}^{2}=\sum_{k=0}^{\infty}\left\|w^{\otimes k}\right\|_{k}^{2}=\sum_{k=0}^{\infty} b_{k}|w|^{2 k}<\infty \quad(w \in \mathbb{B})
$$

by $(3)$, we conclude that $K_{b}(\cdot, w) \in \mathcal{F}_{b}$ for every $w \in \mathbb{B}$. Moreover,

$$
\left[f(\cdot), K_{b}(\cdot, w)\right]_{b}=\sum_{k=0}^{\infty}\left[w^{\otimes k}, \zeta_{k}\right]_{k}=f(w) \quad\left(f \in \mathcal{F}_{b}, w \in \mathbb{B}\right)
$$

by (5). This shows that if $f \in \mathcal{F}_{b}$ and $\left[f(\cdot), K_{b}(\cdot, w)\right]_{b}=0$ for all $w \in \mathbb{B}$, then $f=0$; that is, $\left\{K_{b}(\cdot, w): w \in \mathbb{B}\right\}$ is dense in $\mathcal{F}_{b}$. Also

$$
\left[K_{b}(\cdot, z), K_{b}(\cdot, w)\right]_{b}=\sum_{k=0}^{\infty}\left[w^{\otimes k}, z^{\otimes k}\right]_{k}=\sum_{k=0}^{\infty} b_{k}\langle w, z\rangle^{k}=K_{b}(w, z) \quad(z, w \in \mathbb{B}) .
$$

In particular,

$$
\left\|K_{b}(\cdot, w)\right\|_{b}=\sqrt{K_{b}(w, w)}
$$

We have proved the following.

Theorem 3.3. The space $\mathcal{F}_{b}$ is a reproducing kernel Hilbert space with reproducing kernel $K_{b}(z, w)$.

When $\left\{b_{k}\right\} \in \ell^{1}$, that is, when

$$
\sum_{k=0}^{\infty} b_{k}<\infty
$$

$K_{b}(z, w)$ converges uniformly and absolutely for $(z, w) \in \overline{\mathbb{B}} \times \overline{\mathbb{B}}$. By $(9)$, this implies that an $f \in \mathcal{F}_{b}$ extends continuously to $\overline{\mathbb{B}}$, and thus $\mathcal{F}_{b} \subset A(\mathbb{B})$. If (11) does not hold for a $\left\{b_{k}\right\}$, then $K_{b}$ is unbounded and consequently $\mathcal{F}_{b}$ contains unbounded functions.

The $k$ th Taylor coefficient $\zeta_{k}$ of a given $f \in \mathcal{F}_{b}$ is a finite sum (over $m$ ) of terms of the form $w_{m}^{\otimes k}$ with $w_{m} \in \mathbb{C}^{N}$. Then

$$
\begin{aligned}
f(z) & =\sum_{k=0}^{\infty}\left[z^{\otimes k}, \zeta_{k}\right]_{k}=\sum_{k=0}^{\infty} b_{k} \sum_{m}\left\langle z, w_{m}\right\rangle^{k}=\sum_{k=0}^{\infty} b_{k} \sum_{m} \sum_{|\alpha|=k} \frac{k !}{\alpha !} z^{\alpha} \bar{w}_{m}^{\alpha} \\
& =\sum_{k=0}^{\infty} \sum_{|\alpha|=k}\left(b_{k} \frac{k !}{\alpha !} \sum_{m} \bar{w}_{m}^{\alpha}\right) z^{\alpha}=\sum_{k=0}^{\infty} \sum_{|\alpha|=k} f_{\alpha} z^{\alpha} \quad(z \in \mathbb{B}),
\end{aligned}
$$


where the parentheses give the coefficient $f_{\alpha}$ of $z^{\alpha}$ in terms of the $w_{m}$. Consider now $f=K_{b}(\cdot, w)$ for which $\zeta_{k}=w^{\otimes k}$, a single term, for all $k$. Then

$$
\begin{aligned}
\|f\|_{b}^{2} & =\sum_{k=0}^{\infty}\left\|\zeta_{k}\right\|_{k}^{2}=\sum_{k=0}^{\infty} b_{k}\left\langle w^{\otimes k}, w^{\otimes k}\right\rangle_{k}=\sum_{k=0}^{\infty} b_{k}\langle w, w\rangle^{k}=\sum_{k=0}^{\infty} \sum_{|\alpha|=k} b_{k} \frac{k !}{\alpha !}\left|w^{\alpha}\right|^{2} \\
& =\sum_{k=0}^{\infty} \sum_{|\alpha|=k} b_{k}^{2} \frac{(k !)^{2}}{(\alpha !)^{2}}\left|w^{\alpha}\right|^{2} \frac{\alpha !}{b_{k} k !}=\sum_{k=0}^{\infty} \sum_{|\alpha|=k}\left|f_{\alpha}\right|^{2}\left\|z^{\alpha}\right\|_{b}^{2} .
\end{aligned}
$$

Thus

$$
\left\|z^{\alpha}\right\|_{b}^{2}=\left\|z^{\alpha}\right\|_{k}^{2}=\frac{\alpha !}{b_{|\alpha|}|\alpha| !} .
$$

Consequently, an $f(z)=\sum_{\alpha} f_{\alpha} z^{\alpha} \in H(\mathbb{B})$ belongs to $\mathcal{F}_{b}$ if and only if

$$
\sum_{\alpha} \frac{\left|f_{\alpha}\right|^{2}}{b_{|\alpha|}} \frac{\alpha !}{|\alpha| !}<\infty
$$

The above computation as a byproduct also gives the orthogonality

$$
\left[z^{\alpha}, z^{\beta}\right]_{b}=0 \quad(\alpha \neq \beta)
$$

even when $|\alpha|=|\beta|$ or $|\alpha|=0$. Polarizing the formula for $\|f\|_{b}$, we also obtain an explicit expression for the inner product in $\mathcal{F}_{b}$ in terms of the Taylor coefficients:

$$
[f, g]_{b}=\sum_{k=0}^{\infty} \sum_{|\alpha|=k} f_{\alpha} \bar{g}_{\alpha}\left\|z^{\alpha}\right\|_{b}^{2}=\sum_{k=0}^{\infty} \frac{1}{b_{k} k !} \sum_{|\alpha|=k} \alpha ! f_{\alpha} \bar{g}_{\alpha} .
$$

Proposition 3.4.

$$
\left\{O_{\alpha}^{b}(z)=\sqrt{\frac{b_{|\alpha|}|\alpha| !}{\alpha !}} z^{\alpha}: \alpha \in \mathbb{N}^{N}\right\}
$$

is an orthonormal basis for $\mathcal{F}_{b}$.

Many of the operators we encounter are a class of diagonal operators acting on Dirichlet spaces. We collect their main properties in the next theorem and use them often without mention.

Theorem 3.5. Let $\left\{a_{k}\right\}$ be a sequence of complex numbers and $D: \mathcal{F}_{b} \rightarrow \mathcal{F}_{b}$ be an operator that acts on $f \in \mathcal{F}_{b}$ given by (5) by way of $D f=\sum_{k=0}^{\infty} a_{k} f_{k}$. Then the following conditions hold.

(i) $D$ is a diagonal operator and $D=\sum_{k=0}^{\infty} a_{k} P_{k}$.

(ii) The eigenvalues of $D$ are the $a_{k}$ with eigenspaces $\mathcal{P}_{k}$ of dimension $\delta_{k}$, and $\sigma(D)=\overline{\left\{a_{k}: k \in \mathbb{N}\right\}}$.

(iii) $D$ is invertible if and only if every $a_{k} \neq 0$, and then $D^{-1} f=\sum_{k=0}^{\infty} a_{k}^{-1} f_{k}$.

(iv) $D$ is bounded if and only if $\left\{a_{k}\right\}$ is bounded, and then $\|D\|=\sup _{k}\left\{a_{k}\right\}$. 
(v) $D$ is compact if and only if $a_{k} \rightarrow 0$ as $k \rightarrow \infty$.

(vi) The adjoint of $D$ is given by $D^{*} f=\sum_{k=0}^{\infty} \bar{a}_{k} f_{k}$, so $D$ is self-adjoint if and only if every $a_{k} \in \mathbb{R}$.

(vii) $D$ is positive if and only if every $a_{k} \geq 0$ with $D^{1 / 2} f=\sum_{k=0}^{\infty} \sqrt{a_{k}} f_{k}$.

(viii) The singular values of a compact $D$ are $\left\{\left|a_{k}\right|\right\}$ each of which is repeated $\delta_{k}$ times, and for $0<p<\infty, D$ belongs to $\mathcal{C}^{p}\left(\mathcal{F}_{b}\right)$ if and only if $\left\{a_{k} \delta_{k}^{1 / p}\right\} \in \ell^{p}$ if and only if $\left\{a_{k} k^{(N-1) / p}\right\} \in \ell^{p}$.

Proof. Consider the following order relation $\prec$ on the set of all multi-indices. If $|\alpha|<|\beta|$, let $\alpha \prec \beta$; and among all multi-indices $\alpha$ with the same $|\alpha|$, let $\prec$ be any order, say, the lexicographic order. Note that $f_{k}$ consists of all terms containing $z^{\alpha}$ with the same $|\alpha|=k$ and all these terms are matched with the same $a_{k}$ in $D$. If $f \in \mathcal{F}_{b}$, then $f(z)=\sum_{\alpha} f_{\alpha} z^{\alpha}=\sum_{\alpha} \widetilde{f}_{\alpha} O_{\alpha}^{b}(z)$ and

$$
D f=\sum_{\alpha} a_{|\alpha|} \tilde{f}_{\alpha} O_{\alpha}^{b}
$$

Now almost all the conclusions of the theorem are clear by Proposition 3.4; only for (viii), we note that

$$
\sum_{\alpha}\left|a_{|\alpha|}\right|^{p}=\sum_{k=0}^{\infty}\left|a_{k}\right|^{p} \sum_{|\alpha|=k} 1=\sum_{k=0}^{\infty}\left|a_{k}\right|^{p} \delta_{k} \sim \sum_{k=0}^{\infty}\left|a_{k}\right|^{p} k^{N-1}=\sum_{k=0}^{\infty}\left(\left|a_{k}\right| k^{(N-1) / p}\right)^{p}
$$

using (2).

\section{Dirichlet Spaces}

Our interest in weighted symmetric Fock spaces $\mathcal{F}_{b}$ arises from the well-known spaces corresponding to the special cases of the weight sequence $\left\{b_{k}\right\}$.

Definition 4.1. For $q \in \mathbb{R}$, the Dirichlet space $\mathcal{F}_{q}$ is the reproducing kernel Hilbert space with reproducing kernel $K_{q}(z, w)$ given by $(7)$ when the weight sequence is

$$
b_{k}(q):= \begin{cases}\frac{(1+N+q)_{k}}{k !} & \text { if } q>-(1+N), \\ \frac{k !}{(1-(N+q))_{k}} & \text { if } q \leq-(1+N) .\end{cases}
$$

We use the subscript $q$ instead of $b$ when referring to these spaces, and $q \in \mathbb{R}$ is unrestricted. The first thing to note is that

$$
b_{k}(q) \sim k^{N+q} \quad(k \rightarrow \infty, q \in \mathbb{R})
$$


by (1) so that we have $\lim _{k \rightarrow \infty} b_{k}(q)^{1 / k}=1=: 1 / Z_{q}$ and thus (3) is satisfied. Also for all $q \in \mathbb{R}, b_{0}(q)=1=\|1\|_{q}$, and for $|\alpha|>0$,

$$
\begin{aligned}
\left\|z^{\alpha}\right\|_{q}^{2} & = \begin{cases}\frac{\alpha !}{(1+N+q)_{|\alpha|}} & \text { if } q>-(1+N), \\
\frac{(1-(N+q))_{|\alpha|} \alpha !}{(|\alpha| !)^{2}} & \text { if } q \leq-(1+N),\end{cases} \\
& \sim \frac{1}{|\alpha|^{N+q}} \frac{\alpha !}{|\alpha| !} \quad(q \in \mathbb{R}) .
\end{aligned}
$$

Hence (14) takes the form

$$
\sum_{|\alpha|>0} \frac{\left|f_{\alpha}\right|^{2}}{|\alpha|^{N+q}} \frac{\alpha !}{|\alpha| !}<\infty
$$

as the defining condition for $f(z)=\sum_{\alpha} f_{\alpha} z^{\alpha} \in H(\mathbb{B}) \cap \mathcal{F}_{q}$. By Definition 3.1, every $\mathcal{F}_{q}$ contains the polynomials densely. If $q<-(1+N)$, then $\left\{b_{k}(q)\right\} \in \ell^{1}$ by $(17)$, and by the discussion following $(11), \mathcal{F}_{q} \subset A(\mathbb{B})$.

The reason for considering the particular $b_{k}(q)$ is that special values of $q$ give many well-known spaces. Note that

$$
K_{q}(z, w)= \begin{cases}\frac{1}{(1-\langle z, w\rangle)^{1+N+q}}={ }_{2} F_{1}(1+N+q, 1 ; 1 ;\langle z, w\rangle) & \text { if } q>-(1+N), \\ { }_{2} F_{1}(1,1 ; 1-(N+q) ;\langle z, w\rangle) & \text { if } q \leq-(1+N) .\end{cases}
$$

In particular,

$$
K_{-(1+N)}(z, w)=\frac{1}{\langle z, w\rangle} \log \frac{1}{1-\langle z, w\rangle}
$$

Thus

$$
\mathcal{F}_{q}= \begin{cases}A_{q}^{2} \text { (weighted Bergman spaces) } & \text { if } q>-1, \\ \left.H^{2} \text { (Hardy space }\right) & \text { if } q=-1, \\ \mathcal{A}(\text { Drury-Arveson space }) & \text { if } q=-N, \\ \mathcal{D} \text { (Dirichlet space) } & \text { if } q=-(1+N) .\end{cases}
$$

We use the notation $\|\cdot\|_{\mathcal{A}}$ and $\|\cdot\|_{-N}$ interchangeably, and similarly for the other spaces with special names.

Corollary 4.2. Each $\mathcal{F}_{q}$, and in particular each of $A_{q}^{2}, H^{2}, \mathcal{A}$, and $\mathcal{D}$ is a weighted symmetric Fock space over $\mathbb{C}^{N}$.

Proof. Theorems 3.2 and 3.3 apply.

Note that the case of the Drury-Arveson space $(q=-N)$ is the simplest, because it is unweighted due to $b_{k}(-N)=1$ for all $k$. 
Remark 4.3. There are two immediate critical values of $q$ from the point of view of the $b_{k}(q)$. It is easy to check that $\left\{b_{k}(q)\right\}$ is an increasing sequence for $q \geq-N$, and it is a decreasing sequence for $q<-N$. By contrast, $\left\{b_{k}(q)\right\}$ satisfies $(11)$ and $\mathcal{F}_{q} \subset A(\mathbb{B})$ if and only if $q<-(1+N)$. But $q=-1$ is also critical. By $[12$, Theorem 4.1], there are functions in $A(\mathbb{B})$ that are not in $\mathcal{F}_{b}$ if and only if $q<-1$. We have many more results of this kind.

To obtain one more description of the spaces $\mathcal{F}_{q}$, we recall that the radial derivative of an $f \in H(\mathbb{B})$ given by its homogeneous expansion (5) is $R f=\sum_{k=1}^{\infty} k f_{k}$. The radial derivative is also called the number operator by physicists since it represents the number of particles in a physical system. For all $s, t \in \mathbb{R}$, we define additional radial fractional differential operators $D_{s}^{t}$ of order $t \in \mathbb{R}$ on $H(\mathbb{B})$ by

$$
D_{s}^{t} f:=\sum_{k=0}^{\infty} d_{k}(s, t) f_{k}:=\sum_{k=0}^{\infty} \frac{b_{k}(s+t)}{b_{k}(s)} f_{k} .
$$

These operators are defined by making use of the extra flexibility provided by the parameter $q$ in $b_{k}(q)$ which does not exist in general $\mathcal{F}_{b}$. The coefficients $d_{k}(s, t)$ have uniform growth rate in $\alpha$ with $|\alpha|$ fixed. This particular way of defining the $d_{k}(s, t)$ and thus the $D_{s}^{t}$ in terms of the weight sequence $\left\{b_{k}\right\}$ first appears in [32, Definition 7.1]. Then

$$
D_{q}^{t} K_{q}(z, w)=K_{q+t}(z, w) \quad(q, t \in \mathbb{R}),
$$

where the $D_{q}^{t}$ act on the holomorphic variable $z$.

The $D_{s}^{t}$ clearly commute with each other and with $R$. The essential properties of $D_{s}^{t}$ are that

$$
d_{k}(s, t)>0(k=0,1,2, \ldots) \quad \text { and } \quad d_{k}(s, t) \sim k^{t}(k \rightarrow \infty) .
$$

Other properties are that

$$
D_{s}^{0}=I, \quad D_{s+t}^{u} D_{s}^{t}=D_{s}^{u+t} \quad \text { and } \quad\left(D_{s}^{t}\right)^{-1}=D_{s+t}^{-t}(s, t, u \in \mathbb{R}) .
$$

In particular, $D_{s}^{t}(1)=1$ and $D_{-N}^{1}=I+R$. For comparison, $R$ is not invertible. Many of these claims as well as our next result follow directly from Theorem 3.5.

Proposition 4.4. Every $D_{s}^{t}: \mathcal{F}_{b} \rightarrow \mathcal{F}_{b}$ (and $R$ ) is a positive operator. It is bounded if and only if $t \leq 0$ and compact if and only if $t<0$. The same equivalent conditions applied on the total order characterize the boundedness and compactness of also a composition of several of the $D_{s}^{t}$. Moreover, $D_{s}^{t} \in \mathcal{C}^{p}\left(\mathcal{F}_{q}\right)$ if and only if $N+p t<0$. So $(I+R)^{-1}=D_{1-N}^{-1} \in \mathcal{C}^{p}\left(\mathcal{F}_{q}\right)$ if and only if $p>N$.

Proof. Only the Schatten class membership requires some comment. Now the singular values are $\left|a_{k}\right|=d_{k}(s, t) \sim k^{t}$ and the condition in Theorem 3.5(viii) is that the sequence $\left\{\left(k^{t}\right)^{p} k^{N-1}\right\}$ be summable. 
It is interesting that the conditions in Proposition 4.4 do not depend on $q$. The result about the Schatten class membership of $(I+R)^{-1}$ acting only on $\mathcal{A}$ is given in $[9$, Appendix A] with a more complicated proof.

Corollary 4.5. The map $D_{s}^{t}: \mathcal{F}_{q_{1}} \rightarrow \mathcal{F}_{q_{2}}$ is bounded if and only if $t \leq\left(q_{2}-q_{1}\right) / 2$ and compact if and only if $t<\left(q_{2}-q_{1}\right) / 2$. It belongs to $\mathcal{C}^{p}\left(\mathcal{F}_{q_{1}} \rightarrow \mathcal{F}_{q_{2}}\right)$ if and only if $p>2 N /\left(q_{2}-q_{1}-2 t\right)$. In particular, the inclusion map $i: \mathcal{F}_{q_{1}} \rightarrow \mathcal{F}_{q_{2}}$ is bounded if and only if $q_{1} \leq q_{2}$, compact if and only if $q_{1}<q_{2}$, and belongs to $\mathcal{C}^{p}\left(\mathcal{F}_{q_{1}} \rightarrow \mathcal{F}_{q_{2}}\right)$ if and only if $p>2 N /\left(q_{2}-q_{1}\right)$.

Proof. By Proposition 3.4 and (17), $O_{\alpha}^{q_{1}} \sim k^{\left(q_{1}-q_{2}\right) / 2} O_{\alpha}^{q_{2}}$. Then

$$
D_{s}^{t} f \sim \sum_{\alpha}|\alpha|^{t} \tilde{f}_{\alpha} O_{\alpha}^{q_{1}} \sim \sum_{\alpha}|\alpha|^{t}|\alpha|^{\left(q_{1}-q_{2}\right) / 2} \tilde{f}_{\alpha} O_{\alpha}^{q_{1}} \sim \sum_{k=0}^{\infty} k^{t+\left(q_{1}-q_{2}\right) / 2} f_{k} .
$$

This makes clear the assertions about $D_{s}^{t}$. For $i$, we just set $t=0$.

To form stronger connections with function theoretical techniques, we bring out another characterization of the $\mathcal{F}_{q}$ as Sobolev-type spaces.

Theorem 4.6. Define $I_{s}^{t} f(z):=\left(1-|z|^{2}\right)^{t} D_{s}^{t} f(z)$. An $f \in H(\mathbb{B})$ belongs to $\mathcal{F}_{q}$ if and only if $I_{s}^{t} f$ belongs to $\mathcal{L}_{q}^{2}$ for some $s, t$ satisfying

$$
q+2 t>-1 \text {. }
$$

That is, $I_{s}^{t}: \mathcal{F}_{q} \rightarrow \mathcal{L}_{q}^{2}$ is an imbedding for any $s, t$ satisfying (21). The $\mathcal{L}_{q}^{2}$ norm of any such $I_{s}^{t} f$ and the weighted symmetric Fock space norm $\|f\|_{q}$ are equivalent.

Proof. See [31, Corollary 4.2, Theorem 4.3].

Thus for any $q \in \mathbb{R}, \mathcal{F}_{q}$ is the Besov space $B_{q}^{2}$, where the parameters of $B_{q}^{2}$ follow the pattern of $[31$, p. 386].

Remark 4.7. The equivalent condition stated in Theorem 4.6 for the membership of an $f \in H(\mathbb{B})$ in $\mathcal{F}_{q}$ is

$$
\left\|\left.f\left|\|_{q}^{2}:=C_{q} \int_{\mathbb{B}}\right| D_{s}^{t} f(z)\right|^{2}\left(1-|z|^{2}\right)^{q+2 t} d \nu(z)<\infty\right.
$$

for any $s, t$ satisfying (21). Note that we have a whole family of equivalent norms for each such $s, t$. Polarization gives again equivalent inner products on the $\mathcal{F}_{q}$ :

$$
\llbracket f, g \rrbracket_{q}:=C_{q} \int_{\mathbb{B}} D_{s}^{t} f(z) \overline{D_{s}^{t} g(z)}\left(1-|z|^{2}\right)^{q+2 t} d \nu(z) .
$$

If $q>-1$, then by (21), we can choose $t=0$, no differentiation on $f$ is required in (22), and in such a case $\|f\|_{q}=\|f\|_{\mathcal{L}_{q}^{2}}$. Equivalently, for $q>-1, I_{s}^{t}$ can be taken as the inclusion map and hence $\mathcal{F}_{q} \subset \mathcal{L}_{q}^{2}$. 
The question of which $\mathcal{F}_{q}$ can be characterized by an integral norm of $f$ itself is answered negatively for $q=-N$ in [9, p. 180]. This is extended negatively to all $q<-1$ in $\left[12\right.$, Theorem 4.3] by considering those $\mathcal{F}_{q}$ as Hardy-Sobolev spaces. The answer is clearly positive also for $q=-1$ using the surface measure on $\partial \mathbb{B}$; so in this case $\mathcal{F}_{-1} \subset \mathcal{L}^{2}(\partial \mathbb{B})$.

Membership in $\mathcal{F}_{q}$ can still be decided by an equivalent norm given by an integral of $f$ itself, but only under a limit; see [3, Theorem 9] for $q=-N$ and [4, Proposition 2.1] for $q \geq-(1+N)$.

Corollary 4.8. For any $q, s, t, D_{s}^{t}\left(\mathcal{F}_{q}\right)=\mathcal{F}_{q+2 t}$, and isometrically with appropriate values of the parameters.

Proof. For $f \in \mathcal{F}_{q}$, let $g=D_{s}^{t} f \in \mathcal{F}_{q+2 t}$, and choose $u$ so that $q+2(t+u)>-1$. Then the norm $\|f \mid\|_{q}$ using $D_{s}^{t+u}$ and the norm $\|\mid g\|_{q}$ using $D_{s+t}^{u}$ in (22) are equal. Thus $D_{s}^{t}: \mathcal{F}_{q} \rightarrow \mathcal{F}_{q+2 t}$ is an isometry with the chosen values of the parameters. Throughout we have used (20).

\section{Multipliers and Analytic Hilbert Modules}

A function $g \in H(\mathbb{B})$ is called a multiplier for a Hilbert space $H$ of holomorphic functions on $\mathbb{B}$ if $g f \in H$ whenever $f \in H$. The collection $\mathcal{M}(H)$ of all multipliers of $H$ is a Banach algebra containing $I$. The natural norm $\|\cdot\|_{\mathcal{M}(H)}$ on $\mathcal{M}(H)$ is the norm of the operator $M_{g}: H \rightarrow H$ of multiplication by $g$.

We have $\mathcal{M}\left(\mathcal{F}_{b}\right) \subset \mathcal{F}_{b}$ for all $b$ since $1 \in \mathcal{F}_{b}$. Also, since $\|1\|_{b}=1$, if $g \in \mathcal{M}\left(\mathcal{F}_{b}\right)$, then $\|g\|_{b}=\|g 1\|_{b}=\left\|M_{g} 1\right\| \leq\left\|M_{g}\right\|\|1\|_{b}=\left\|M_{g}\right\|=\|g\|_{\mathcal{M}\left(\mathcal{F}_{b}\right)}$. Further, by $[12$, Lemmas 5.1 and 5.2] (and by [9, Proposition 2.2] for the case $\mathcal{F}_{-N}$ ), $\mathcal{M}\left(\mathcal{F}_{b}\right) \subset H^{\infty}$ and $\|g\|_{H^{\infty}} \leq\|g\|_{\mathcal{M}\left(\mathcal{F}_{b}\right)}$ for $g \in \mathcal{M}\left(\mathcal{F}_{b}\right)$. The reverse inequalities in the last two sentences are not true for all $b$ however. In any case, $\mathcal{M}\left(\mathcal{F}_{b}\right) \subset \mathcal{F}_{b} \cap H^{\infty}$.

Moreover, the well-known quick computation

$$
\left[f(\cdot), M_{g}^{*} K_{b}(\cdot, w)\right]_{b}=\left[(g f)(\cdot), K_{b}(\cdot, w)\right]_{b}=g(w) f(w)=\left[f(\cdot), \overline{g(w)} K_{b}(\cdot, w)\right]_{b}
$$

for any $w \in \mathbb{B}$ and $f \in \mathcal{F}_{b}$ shows that

$$
M_{g}^{*} K_{b}(\cdot, w)=\overline{g(w)} K_{b}(\cdot, w),
$$

that is, $\overline{g(w)}$ for $w \in \mathbb{B}$ is an eigenvalue of $M_{g}^{*}$ with eigenvector $K_{b}(\cdot, w)$.

It is clear by the $t=0$ case of Remark 4.7 that $\mathcal{M}\left(\mathcal{F}_{q}\right)=H^{\infty}$ for $q \geq-1$ with equal norms. But for other values of $q$, the inclusion of the multiplier algebra in $H^{\infty}$ is proper. By [12, Theorem 4.2], for $q<-1$, there are functions in $A(\mathbb{B})$ that are not in $\mathcal{M}\left(\mathcal{F}_{q}\right)$.

If $q<-(1+N)$, then $\mathcal{F}_{q}$ an algebra and $\mathcal{M}\left(\mathcal{F}_{q}\right)=\mathcal{F}_{q}$; see [36, Theorem 3.8]. For $-(1+N) \leq q<-1$, characterizations of $\mathcal{M}\left(\mathcal{F}_{q}\right)$ are not so simple. See, for example, [36, Theorems 3.7 and 3.10].

The spaces $\mathcal{F}_{b}$ include all holomorphic polynomials in $N$ variables densely, and these polynomials are multipliers for all $\mathcal{F}_{b}$. Thus each $\mathcal{F}_{b}$ is a module over the polynomial algebra $\mathbb{C}[z]=\mathbb{C}\left[z_{1}, \ldots, z_{N}\right]$. 
The following definitions are taken from [17, Sec. 2.2].

Definition 5.1. A point $w \in \mathbb{C}^{N}$ is called a virtual point of a reproducing kernel Hilbert space $H$ of holomorphic functions on $\mathbb{B}$ if the point evaluation functional at $w \in \mathbb{B}$ extends boundedly from $\mathbb{C}[z]$ to all of $H$. The space $H$ is called an analytic Hilbert module if $1 \in H$, polynomials are dense in $H$, the multiplier algebra $\mathcal{M}(H)$ of $H$ contains $\mathbb{C}[z]$, and the set of virtual points of $H$ coincides with $\mathbb{B}$.

Theorem 5.2. The space $\mathcal{F}_{b}$ is an analytic Hilbert module if and only if $\left\{b_{k}\right\} \notin \ell^{1}$.

Proof. We only have to check the virtual points. We know that points of $\mathbb{B}$ are virtual points by Theorem 3.2.

First suppose $\left\{b_{k}\right\} \notin \ell^{1}$, and that $z_{0} \in \mathbb{C}^{N}$ with $\left|z_{0}\right| \geq 1$ is a virtual point of $\mathcal{F}_{b}$. Then there is a $C$ depending on $z_{0}$ such that $\left|K_{b}\left(z_{0}, w\right)\right| \leq C\left\|K_{b}(\cdot, w)\right\|_{b}$ for all small $w$, that is,

$$
\left|\sum_{k=0}^{\infty} b_{k}\left\langle z_{0}, w\right\rangle^{k}\right|^{2} \leq C \sum_{k=0}^{\infty} b_{k}|w|^{2 k} \quad\left(|w|<1 /\left|z_{0}\right|\right)
$$

by (10). Let $w=t z_{0}$ with $t \geq 0$ so small that $t^{2}<t$; then

$$
\left(\sum_{k=0}^{\infty} b_{k} t^{k}\left|z_{0}\right|^{2 k}\right)^{2} \leq C \sum_{k=0}^{\infty} b_{k} t^{2 k}\left|z_{0}\right|^{2 k} \leq C \sum_{k=0}^{\infty} b_{k} t^{k}\left|z_{0}\right|^{2 k} \quad\left(0 \leq t<\frac{1}{\left|z_{0}\right|^{2}}\right) .
$$

After an obvious cancellation and letting $t \rightarrow 1 /\left|z_{0}\right|^{2}$ from the left, we obtain

$$
\sum_{k=0}^{\infty} b_{k} \leq C
$$

contrary to assumption. Thus $z_{0}$ cannot be a virtual point of $\mathcal{F}_{b}$.

Now suppose $\left\{b_{k}\right\} \in \ell^{1}$, and let $z_{0} \in \mathbb{C}^{N}$ with $\left|z_{0}\right|=1$. Now there is a $C$ depending on $z_{0}$ such that $\left|K_{b}\left(z_{0}, w\right)\right| \leq C$ for all $w \in \mathbb{B}$. On the other hand,

$$
1 \leq K_{q}(w, w)=\sum_{k=0}^{\infty} b_{k}|w|^{2} \leq C \quad(w \in \mathbb{B})
$$

since $b_{k}>0$ with $b_{0}=1$. Then $\left|K_{b}\left(z_{0}, w\right)\right| \leq C \sqrt{K_{b}(w, w)}=C\left\|K_{b}(\cdot, w)\right\|_{q}$ for all $w \in \mathbb{B}$. Using the fact that the functions $K_{q}(\cdot, w)$ are dense in $\mathcal{F}_{q}$, we conclude that $z_{0}$ is a virtual point of $\mathcal{F}_{b}$.

The proof of the next result is clear by Remark 4.3. The case $q=-N$ is in $[17$, p. 128].

Corollary 5.3. The Dirichlet spaces $\mathcal{F}_{q}$ are analytic Hilbert modules if and only if $q \geq-(1+N)$. 


\section{Shift Operators}

The importance of shift operators acting on function spaces cannot be overemphasized in operator theory. Here we develop the essential properties of the multivariable shift operator on the spaces $\mathcal{F}_{b}$ and $\mathcal{F}_{q}$ realized as multiplications by the coordinate variables of $\mathbb{C}^{N}$.

Notation 6.1. If $T=\left(T_{1}, \ldots, T_{N}\right)$ is an $N$-tuple of operators on a Hilbert space $H$, for convenience we write $T^{*}:=\left(T_{1}^{*}, \ldots, T_{N}^{*}\right)$ and use the short-hand notation

$$
T T^{*}:=T_{1} T_{1}^{*}+\cdots+T_{N} T_{N}^{*} \quad \text { and } \quad T^{*} T:=T_{1}^{*} T_{1}+\cdots+T_{N}^{*} T_{N} .
$$

Warning 6.2. In many other contexts, $T^{*} T$ represents a matrix of operators, but we do not mean that. What is meant by $T^{*} T$ here is just the single operator defined by the sum in Notation 6.1 .

Definition 6.3. Let $H$ be a Hilbert space containing $\mathbb{C}[z]$ and let $S_{j}: H \rightarrow H$ be the operator of multiplication by $z_{j}, j=1, \ldots, N$. The operator $S=\left(S_{1}, \ldots, S_{N}\right)$ is called the (forward, unilateral) shift operator on $H$. When $H=\mathcal{F}_{b}$, we write $S_{b}$ for $S$ and call it the b-shift, and when $H=\mathcal{F}_{q}$, we write $S_{q}$ for $S$ and call it the $q$-shift.

The operator of multiplication $M_{p}$ by $p \in \mathbb{C}[z]$ defined on $H$ can also be expressed as the operator $p(S)=p\left(S_{1}, \ldots, S_{N}\right)$.

Let $S_{b_{j}}^{*}$ be the adjoint of $S_{b_{j}}$. Then $S_{b_{j}} z^{\alpha}=z^{\alpha+e_{j}}$, and $S_{b_{j}}^{*} z^{\alpha}=C_{j} z^{\alpha-e_{j}}$ for $\alpha_{j}>0$ by the uniqueness of the adjoint. Clearly $S_{b_{j}}^{*} z^{\alpha}=0$ if $\alpha_{j}=0$ and so $S_{b_{j}}^{*} 1=0$. To find $C_{j}$, we compute

$$
\begin{aligned}
{\left[S_{b_{j}}^{*} z^{\alpha}, z^{\alpha-e_{j}}\right]_{b} } & =\left[z^{\alpha}, S_{b_{j}} z^{\alpha-e_{j}}\right]_{b}=\left[z^{\alpha}, z^{\alpha}\right]_{b}=\left\|z^{\alpha}\right\|_{b}^{2} \\
& =\left[C_{j} z^{\alpha-e_{j}}, z^{\alpha-e_{j}}\right]_{b}=C_{j}\left\|z^{\alpha-e_{j}}\right\|_{b}^{2}
\end{aligned}
$$

Hence by (13),

$$
S_{b_{j}}^{*} z^{\alpha}=C_{j} z^{\alpha-e_{j}}=\frac{\left\|z^{\alpha}\right\|_{b}^{2}}{\left\|z^{\alpha-e_{j}}\right\|_{b}^{2}} z^{\alpha-e_{j}}=\frac{\alpha_{j}}{|\alpha|} \frac{b_{|\alpha|-1}}{b_{|\alpha|}} z^{\alpha-e_{j}} \quad(|\alpha|>0) .
$$

Apparently the $S_{b_{j}}$ commute with each other, and so do the $S_{b_{j}}^{*}$, but $S_{b_{j}}$ does not commute with $S_{b_{j}}^{*}$. Then using Notation $6.1, S_{b} S_{b}^{*} 1=0$,

$$
S_{b} S_{b}^{*} z^{\alpha}=\frac{b_{|\alpha|-1}}{b_{|\alpha|}} z^{\alpha} \quad(|\alpha|>0) \quad \text { and } \quad S_{b}^{*} S_{b} z^{\alpha}=\frac{N+|\alpha|}{1+|\alpha|} \frac{b_{|\alpha|}}{b_{|\alpha|+1}} z^{\alpha} \quad(|\alpha| \geq 0) .
$$

Note that the coefficients of $z^{\alpha}$ in $S_{b} S_{b}^{*}$ and $S_{b}^{*} S_{b}$ depend on $|\alpha|$ and not on the components of $\alpha$. Thus if $f \in \mathcal{F}_{b}$ is given by its homogeneous expansion (5), then

$$
S_{b} S_{b}^{*} f=\sum_{k=1}^{\infty} \frac{b_{k-1}}{b_{k}} f_{k} \quad \text { and } \quad S_{b}^{*} S_{b} f=\sum_{k=0}^{\infty} \frac{N+k}{1+k} \frac{b_{k}}{b_{k+1}} f_{k} .
$$


Theorem 3.5 immediately implies the following. The extra conditions on $b$ are not vacuous by [40, Example 3.35].

Proposition 6.4. The operators $S_{b} S_{b}^{*}$ and $S_{b}^{*} S_{b}$ are positive. They are bounded if and only the sequence $\left\{b_{k} / b_{k+1}\right\}$ is bounded, in which case $S_{b_{j}}$ and $S_{b}$ are also bounded along with their adjoints. Also $\left\|S_{b} S_{b}^{*}\right\|=\sup \left\{b_{k} / b_{k+1}\right\}$ and a counterpart for $\left\|S_{b}^{*} S_{b}\right\|$ holds. Further, $S_{b} S_{b}^{*}$ and $S_{b}^{*} S_{b}$ are compact if and only if $b_{k} / b_{k+1} \rightarrow 0$ as $k \rightarrow \infty$, which also implies the compactness of $S_{b_{j}}^{*}$ and $S_{b}^{*}$. Moreover, $S_{b} S_{b}^{*}$ and $S_{b}^{*} S_{b}$ belong to $\mathcal{C}^{p}\left(\mathcal{F}_{b}\right)$ if and only if $\left\{\left(b_{k} / b_{k+1}\right) k^{(N-1) / p}\right\} \in \ell^{p}$. Finally, $S_{b}^{*} S_{b}$ is invertible, but $S_{b} S_{b}^{*}$ is not.

Continuing,

$$
\begin{aligned}
& \left(I-S_{b} S_{b}^{*}\right) f=f_{0}+\sum_{k=1}^{\infty}\left(1-\frac{b_{k-1}}{b_{k}}\right) f_{k} \\
& \left(I-S_{b}^{*} S_{b}\right) f=\sum_{k=0}^{\infty}\left(1-\frac{N+k}{1+k} \frac{b_{k}}{b_{k+1}}\right) f_{k} .
\end{aligned}
$$

So unless $\left\{b_{k}\right\}$ is a constant sequence, the operator $I-S_{b} S_{b}^{*}$ is not the projection onto the one-dimensional space of constants in $\mathcal{F}_{b}$. The coefficients are all positive if and only if $\left\{b_{k}\right\}$ is a increasing sequence. Even if $b_{m}<b_{m-1}$ for one $m>0$, substituting $f(z)=z_{1}^{m}$ to the above equation yields $\left[\left(I-S_{b} S_{b}^{*}\right) f, f\right]_{b}<0$. In such a case,

$$
\lambda=\frac{b_{m}-b_{m-1}}{b_{m}}<0
$$

is a negative eigenvalue with eigenvector $z_{1}^{m}$ of $I-S_{b} S_{b}^{*}$. Now the following results are also direct implications of Theorem 3.5.

Proposition 6.5. The operator $I-S_{b} S_{b}^{*}$ is positive if and only if $\left\{b_{k}\right\}$ is an increasing sequence. Moreover, $I-S_{b} S_{b}^{*}=1 \otimes 1$ if and only if $\left\{b_{k}\right\}$ is a constant sequence with the constant equal to $b_{0}=1$.

Proposition 6.6. The operator $I-S_{b}^{*} S_{b}$ is positive if and only if $\left\{b_{k}\right\}$ is a sequence that increases initially fast in the sense that $(1+k) b_{k+1} \geq(N+k) b_{k}$ which reduces to increasing for $N=1$.

Definition 6.7. A row contraction on a Hilbert space $H$ is a commuting $N$-tuple of operators $T=\left(T_{1}, \ldots, T_{N}\right)$ on $H$ satisfying

$$
\left\|T_{1} u_{1}+\cdots+T_{N} u_{N}\right\|_{H}^{2} \leq\left\|u_{1}\right\|_{H}^{2}+\cdots+\left\|u_{N}\right\|_{H}^{2}
$$


for all $u_{1}, \ldots, u_{N} \in H$. The norm of a Hilbert space containing polynomials in $N$ complex variables is called contractive if the associated shift is a row contraction and the constants are orthogonal to all polynomials vanishing at 0 .

Note that if $T$ is a row contraction, then any individual $T_{j}$ is a contraction, but the converse of this statement need not be true. By [9, Remark 3.2], $T$ is a row contraction if and only if $I-T T^{*}$ is a positive operator.

Proposition 6.8. The b-shift $S_{b}$ is a row contraction and $\|\cdot\|_{b}$ is a contractive norm if and only if $\left\{b_{k}\right\}$ is increasing. In such a case, $\|\cdot\|_{b} \leq\|\cdot\|_{\mathcal{A}}$. The adjoint $S_{b}^{*}$ of the b-shift is a row contraction if and only if $\left\{b_{k}\right\}$ is increasing initially fast in the sense described in Proposition 6.6.

Proof. For the first statement, we combine the previous paragraph with Propositions 6.5 and 3.4. For the second, we use [9, Theorem 4.3], which also implies $b_{k} \geq 1=b_{0}$, which is obvious for an increasing $\left\{b_{k}\right\}$. For the third, we use Proposition 6.6 instead.

We use Theorem 3.5 one more time to conclude the following.

Proposition 6.9. The operator $I-S_{b} S_{b}^{*}$ is compact if and only if $b_{k} / b_{k+1} \rightarrow 1$ as $k \rightarrow \infty$, and $I-S_{b} S_{b}^{*} \in \mathcal{C}^{p}\left(\mathcal{F}_{b}\right)$ if and only if $\left\{\left(1-b_{k} / b_{k+1}\right) k^{(N-1) / p}\right\} \in \ell^{p}$. Similar results hold for $I-S_{b}^{*} S_{b}$.

Specializing to $H=\mathcal{F}_{q}$, by Definition 4.1 and $(24)$, we have $S_{q_{j}}^{*} 1=0$, and

$$
S_{q_{j}}^{*} z^{\alpha}= \begin{cases}\frac{\alpha_{j}}{N+q+|\alpha|} z^{\alpha-e_{j}} & \text { if } q>-(1+N), \\ \frac{(-(N+q)+|\alpha|) \alpha_{j}}{|\alpha|^{2}} z^{\alpha-e_{j}} & \text { if } q \leq-(1+N),\end{cases}
$$

for $|\alpha|>0$, meaning that $S_{q_{j}}^{*} z^{\alpha}=0$ if $\alpha_{j}=0$. Also

$$
\begin{aligned}
& S_{q} S_{q}^{*} z^{\alpha}= \begin{cases}\frac{|\alpha|}{N+q+|\alpha|} z^{\alpha} & \text { if } q>-(1+N), \\
\frac{-(N+q)+|\alpha|}{|\alpha|} z^{\alpha} & \text { if } q \leq-(1+N),\end{cases} \\
& S_{q}^{*} S_{q} z^{\alpha}= \begin{cases}\frac{N+|\alpha|}{1+N+q+|\alpha|} z^{\alpha} & \text { if } q>-(1+N), \\
\frac{(1-(N+q)+|\alpha|)(N+|\alpha|)}{(1+|\alpha|)^{2}} z^{\alpha} & \text { if } q \leq-(1+N),\end{cases}
\end{aligned}
$$


and

$$
\begin{aligned}
& S_{q} S_{q}^{*} f= \begin{cases}\sum_{k=1}^{\infty} \frac{k}{N+q+k} f_{k}, & \text { if } q>-(1+N), \\
\sum_{k=1}^{\infty} \frac{-(N+q)+k}{k} f_{k}, & \text { if } q \leq-(1+N),\end{cases} \\
& S_{q}^{*} S_{q} f= \begin{cases}\sum_{k=0}^{\infty} \frac{N+k}{1+N+q+k} f_{k}, & \text { if } q>-(1+N), \\
\sum_{k=0}^{\infty} \frac{(1-(N+q)+k)(N+k)}{(1+k)^{2}} f_{k}, & \text { if } q \leq-(1+N) .\end{cases}
\end{aligned}
$$

The coefficient of $f_{k}$ in $S_{q} S_{q}^{*} f$ is increasing in $k$ if $q \geq-N$ and decreasing otherwise. The coefficient of $f_{k}$ in $S_{q}^{*} S_{q} f$ is increasing in $k$ if $q \geq-1$ and decreasing otherwise. Thus

$$
\left\|S_{q} S_{q}^{*}\right\|= \begin{cases}1 & \text { if } q \geq-N, \\ 1 /(1+N+q) & \text { if }-(1+N)<q<-N, \\ 1-(N+q) & \text { if } q \leq-(1+N),\end{cases}
$$

and

$$
\left\|S_{q}^{*} S_{q}\right\|= \begin{cases}1 & \text { if } q \geq-1, \\ N /(1+N+q) & \text { if }-(1+N)<q<-1, \\ N(1-(N+q)) & \text { if } q \leq-(1+N) .\end{cases}
$$

In particular, every $S_{q_{j}}$ and every $S_{q}$ are bounded operators along with their adjoints. The results for $S_{q} S_{q}^{*}$ appear in [6, Corollary 3.7 and Theorem 4.1] for Dirichlet spaces on bounded symmetric domains restricted to the cases counterpart to $q>-(1+N)$. The result for $S_{-N}^{*} S_{-N}$ is in [9, Proposition 5.3]. Hence $\lim _{q \rightarrow-\infty}\left\|S_{q} S_{q}^{*}\right\|=\lim _{q \rightarrow-\infty}\left\|S_{q}^{*} S_{q}\right\|=\infty$. The more interesting observation is that $\lim _{q \rightarrow-(1+N)^{+}}\left\|S_{q} S_{q}^{*}\right\|=\lim _{q \rightarrow-(1+N)^{+}}\left\|S_{q}^{*} S_{q}\right\|=\infty$ while $\left\|S_{-(1+N)} S_{-(1+N)}^{*}\right\|=2$ and $\left\|S_{-(1+N)}^{*} S_{-(1+N)}\right\|=2 N$.

It is not difficult to compute the precise norms of the individual $S_{q_{j}}$, and in fact of any $S_{q}^{\beta}$. First

$$
\left\|S_{q_{j}} f\right\|_{q}^{2}=\sum_{\alpha}\left|f_{\alpha}\right|^{2}\left\|z^{\alpha+e_{j}}\right\|_{q}^{2}=\sum_{\alpha}\left|f_{\alpha}\right|^{2}\left\|z^{\alpha}\right\|_{q}^{2} \mu_{\alpha}(j),
$$

where

$$
\mu_{\alpha}(j)= \begin{cases}\frac{1+\alpha_{j}}{1+N+q+|\alpha|} & \text { if } q>-(1+N), \\ \frac{1-(N+q)+|\alpha|}{1+|\alpha|} \frac{1+\alpha_{j}}{1+|\alpha|} & \text { if } q \leq-(1+N)\end{cases}
$$


by (18). For $q \geq-N$, always $\mu_{\alpha}(j) \leq 1$, we consider $f(z)=z_{j}^{\alpha_{j}}$, and let $\alpha_{j} \rightarrow \infty$. For $q<-N, \mu_{\alpha}(j)$ is largest when $\alpha=(0, \ldots, 0)$, and we consider $f(z)=1$. Then we obtain

$$
\left\|S_{q_{j}}\right\|= \begin{cases}1 & \text { if } q \geq-N \\ 1 / \sqrt{1+N+q} & \text { if }-(1+N)<q<-N \\ \sqrt{1-(N+q)} & \text { if } q \leq-(1+N),\end{cases}
$$

where the critical values of $q$ are those of $(29)$. Thus the individual shifts on $\mathcal{F}_{q}$ are contractions if and only if $q \geq-N$, and this range includes the Drury-Arveson space. Again $\lim _{q \rightarrow-(1+N)+}\left\|S_{q_{j}}\right\|=\infty$ while $\left\|S_{-(1+N)_{j}}\right\|=\sqrt{2}$. Letting $p(z)=z^{\beta}$ and arguing similarly, we also see that

$$
\left\|S_{q}^{\beta}\right\|= \begin{cases}\frac{\sqrt{\beta !}}{\sqrt{(1+N+q)_{|\beta|}}} & \text { if } q \geq-N, \\ \frac{\sqrt{\beta !(1-(N+q))_{|\beta|}}}{|\beta| !} & \text { if } q \leq-(1+N)<q<-N,\end{cases}
$$

From Proposition 6.4, we obtain our next result.

Corollary 6.10. The operators $S_{q} S_{q}^{*}$ and $S_{q}^{*} S_{q}$ are never compact.

We also have

$$
\left(I-S_{q} S_{q}^{*}\right) f= \begin{cases}f_{0}+\sum_{k=1}^{\infty} \frac{N+q}{N+q+k} f_{k} & \text { if } q>-(1+N), \\ f_{0}+\sum_{k=1}^{\infty} \frac{N+q}{k} f_{k} & \text { if } q \leq-(1+N)\end{cases}
$$

and

$$
\left(I-S_{q}^{*} S_{q}\right) f= \begin{cases}\sum_{k=0}^{\infty} \frac{1+q}{1+N+q+k} f_{k} & \text { if } q>-(1+N), \\ \sum_{k=0}^{\infty} \frac{1+N(-1+N+q)+(1+q) k}{(1+k)^{2}} f_{k} & \text { if } q \leq-(1+N) .\end{cases}
$$

The following is now clear by Remark 4.3 and Proposition 6.5.

Corollary 6.11. The operator $I-S_{q} S_{q}^{*}$ is positive, equivalently $S_{q}$ is a row contraction, equivalently $\|\cdot\|_{q}$ is a contractive norm, if and only if $q \geq-N$. In such a case, $\|\cdot\|_{q} \leq\|\cdot\|_{-N}$. Further, $I-S_{q} S_{q}^{*}$ is of finite rank and in such a case equals $1 \otimes 1$ if and only if $q=-N$, that is, only for the Drury-Arveson space $\mathcal{F}_{-N}$. If $q \neq-N$, then $I-S_{q} S_{q}^{*}$ is invertible.

In fact, for $q>-N$ and $|\alpha|>0,\left\|z^{\alpha}\right\|_{q}<\left\|z^{\alpha}\right\|_{-N}$ as can be seen from (18). For $q<-N$, the explicit forms of $\left\|z^{\alpha}\right\|_{q}^{2}$ in (18) show that $\left\|z^{\alpha}\right\|_{q}>\left\|z^{\alpha}\right\|_{-N}$ for 
$|\alpha|>0$, and hence for all $f \in \mathcal{F}_{q}$ except for constants. This observation combined with Corollary 4.5 implies that within the scale of spaces $\mathcal{F}_{q}$, the Drury-Arveson space $\mathcal{A}(q=-N)$ is the smallest one with a contractive norm. This should be compared to the fact that within the same scale, this space is the largest one with a complete Nevanlinna-Pick kernel; see [2, Theorem 7.33 and Corollary 7.41].

Definition 6.12. An $N$-tuple $T=\left(T_{1}, \ldots, T_{N}\right)$ of operators is called a spherical isometry if $I-T^{*} T=0$, a spherical contraction if $I-T^{*} T \geq 0$, and a spherical expansion if $I-T^{*} T \leq 0$, where we use Notation 6.1.

Corollary 6.13. The $N$-tuple $S_{q}$ is a spherical contraction if and only if $q \geq-1$, and it is a spherical expansion if and only if $q \leq-1$. It is a spherical isometry if and only if $q=-1$, that is, only on the Hardy space $\mathcal{F}_{-1}$. If $q \neq-1$, then $I-S_{q}^{*} S_{q}$ is invertible.

Proof. Consider (31). If $q>-(1+N)$, positivity of $I-S_{q}^{*} S_{q}$ holds only for $q \geq-1$. If $q \leq-(1+N)$, then $-1+N+q \leq-2$ and the coefficient of $f_{0}$ is $1+N(-1+N+q) \leq 1-2 N<0$; so there is no positivity for $q \leq-(1+N)$.

Corollary 6.14. Both operators $I-S_{q} S_{q}^{*}$ and $I-S_{q}^{*} S_{q}$ are always compact, and they belong to $\mathcal{C}^{p}\left(\mathcal{F}_{q}\right)$ if and only if $\left\{k^{(N-1-p) / p}\right\} \in \ell^{p}$ if and only if $p>N$.

The condition $p>N$ in Corollary 6.14 and Proposition 4.4 is the same, because the operators $(I+R)^{-1}, I-S_{q} S_{q}^{*}$, and $I-S_{q}^{*} S_{q}$ have their coefficient multiplier sequence $\sim 1 / k$.

The formulas for $S_{q} S_{q}^{*}$ and $S_{q}^{*} S_{q}$ bear a strong resemblance to the definitions of the $D_{s}^{t}$ and $R$, and indeed we are able to write them in terms of simple combinations of our radial differential operators. It is easy to check that

$$
\begin{aligned}
S_{q} S_{q}^{*} & = \begin{cases}\frac{1}{N+q} D_{q}^{-1} R & \text { if } q>-N, \\
-(N+q)\left(R^{\prime}\right)^{-1} D_{q}^{1} & \text { if } q \leq-(2+N),\end{cases} \\
& = \begin{cases}(R+(N+q) I)^{-1} R & \text { if } q>-(1+N), \\
(R-(N+q) I)\left(R^{\prime}\right)^{-1} & \text { if } q \leq-(1+N),\end{cases}
\end{aligned}
$$

where $R^{\prime}$ represents the restriction of $R$ to $\mathcal{F}_{q} / \mathbb{C}$ on which it is invertible with inverse

$$
\left(R^{\prime}\right)^{-1}=(1 \otimes 1+R)^{-1}-1 \otimes 1
$$

and

$$
S_{q}^{*} S_{q}= \begin{cases}\frac{N}{1+N+q} D_{-1}^{1} D_{1+q}^{-1} & \text { if } q>-(1+N), \\ N(1-(N+q)) D_{-1}^{1} D_{-1+q}^{1}\left(D_{-N}^{-1}\right)^{2} & \text { if } q \leq-(1+N) .\end{cases}
$$


A formula for $\left(S_{q}^{*} S_{q}\right)^{-1}$ can be written using (20), but we have no use for it. Similar formulas can be tried for the other operators as well.

\section{7. von Neumann Inequalities}

We are now ready to prove a von Neumann inequality with respect to every weighted symmetric Fock space $\mathcal{F}_{b}$ for row contractions on arbitrary Hilbert spaces. It turns out that among the scale of spaces $\mathcal{F}_{q}$, the inequality with respect to the DruryArveson space $\mathcal{A}$ is the simplest one, though not always the sharpest one.

Definition 7.1. Let $T=\left(T_{1}, \ldots, T_{N}\right)$ be a row contraction on a Hilbert space $H$. Its defect operator is $\Delta_{T}=\left(I-T T^{*}\right)^{1 / 2}$, which is the unique positive square root.

Clearly $\Delta_{S_{b}} 1=1$ for any $b$. The computations in Sec. 6 show that

$$
\begin{aligned}
& \Delta_{S_{b}} f=f_{0}+\sum_{k=1}^{\infty} \sqrt{1-\frac{b_{k-1}}{b_{k}}} f_{k} \quad\left(\left\{b_{k}\right\} \text { is increasing }\right), \\
& \Delta_{S_{q}} f=f_{0}+\sum_{k=1}^{\infty} \sqrt{\frac{N+q}{N+q+k}} f_{k} \quad(q \geq-N) .
\end{aligned}
$$

An operator tuple $T=\left(T_{1}, \ldots, T_{N}\right)$ on $H$ gives rise to a map $J_{T}: \mathcal{B}(H) \rightarrow \mathcal{B}(H)$ defined by

$$
J_{T}(A):=T_{1} A T_{1}^{*}+\cdots+T_{N} A T_{N}^{*} \quad(A \in \mathcal{B}(H)) .
$$

If $T$ is a row contraction, then $I \geq J_{T}(I)=T T^{*} \geq J_{T}^{2}(I) \geq \cdots \geq 0$ using Notation 6.1. Thus $\lim _{n \rightarrow \infty} J_{T}^{n}(I)=T_{\infty}$ exists in the strong operator topology and satisfies $0 \leq T_{\infty} \leq I$.

Definition 7.2. If $T_{\infty}=0$ for a row contraction $T$ on a Hilbert space $H$, then $T$ is called null (or pure).

If $T T^{*} \leq r I$ with $0<r<1$, then $T$ is clearly null.

When $H=\mathcal{F}_{b}$ and $T=S_{b}$, the $b$-shift, a straightforward computation using (25) repeatedly shows that

$$
J_{S_{b}}^{m}(I) z^{\alpha}=\frac{b_{|\alpha|-m}}{b_{|\alpha|}} z^{\alpha} \quad(|\alpha| \geq m) \quad \text { and } \quad J_{S_{b}}^{m}(I) f=\sum_{k=m}^{\infty} \frac{b_{k-m}}{b_{k}} f_{k}
$$

for $f \in \mathcal{F}_{b}$ with the homogeneous expansion (5); also $J_{S_{b}}^{m}(I) p=0$ if $p$ is a polynomial of degree less than $m$. In particular,

$$
J_{S_{q}}^{m}(I) f= \begin{cases}\sum_{k=m}^{\infty} \frac{(1+k-m)_{m}}{(1+N+q+k-m)_{m}} f_{k} \quad \text { if } q>-(1+N), \\ \sum_{k=m}^{\infty} \frac{(1-(N+q)+k-m)_{m}}{(1+k-m)_{m}} f_{k} \quad \text { if } q \leq-(1+N) .\end{cases}
$$


If $S_{b}$ is a row contraction which happens if and only if $\left\{b_{k}\right\}$ is increasing by Proposition 6.8 , then the coefficients of the $f_{k}$ in $J_{S_{b}}^{m}(I) f$ are all $\leq 1$, and

$$
\left\|J_{S_{b}}^{m}(I) f\right\|_{b}^{2}=\sum_{k=m}^{\infty} \frac{b_{k-m}^{2}}{b_{k}^{2}}\left\|f_{k}\right\|_{k}^{2} \leq \sum_{k=m}^{\infty}\left\|f_{k}\right\|_{k}^{2} \rightarrow 0 \quad \text { as } m \rightarrow \infty,
$$

because it is the tail end of the series in (6). Thus $\left(S_{b}\right)_{\infty}=0$ for $S_{b}$ a row contraction. In particular, this holds for $S_{q}$ when $q \geq-N$.

On the other hand, by first replacing $k-m$ by $k$, it is easy to see that

$$
\left\|J_{S_{q}}^{m}(I) f\right\|_{q}^{2} \sim m^{-2(N+q)} \sum_{k=m}^{\infty}\left\|f_{k}\right\|_{q}^{2} .
$$

For $q<-N$, the power on $m$ is strictly positive. For such $q$, define

$$
\widetilde{f}(z)=\sum_{k=1}^{\infty} \frac{1}{k^{(1-2(N+q)) / 2}} z_{1}^{k}
$$

Then by (18),

$$
\|\widetilde{f}\|_{q}^{2} \sim \sum_{k=1}^{\infty} \frac{1}{k^{1-2(N+q)}} \frac{1}{k^{N+q}}<\infty
$$

that is, $f \in \mathcal{F}_{q}$. But

$$
\sum_{k=m}^{\infty}\left\|\tilde{f}_{k}\right\|_{q}^{2}=\sum_{k=m}^{\infty} \frac{1}{k^{1-(N+q)}} \sim \int_{m}^{\infty} \frac{d x}{x^{1-(N+q)}} \sim \frac{1}{m^{-(N+q)}} .
$$

Thus $\left\|J_{S_{q}}^{m}(I) \tilde{f}\right\|_{q}^{2} \sim m^{-(N+q)} \rightarrow \infty$ as $m \rightarrow \infty$.

The following result emerges by collecting the pieces together.

Proposition 7.3. The b-shift $S_{b}$ is null whenever it is a row contraction. In particular, this holds for every $q$-shift $S_{q}$ with $q \geq-N$. Conversely, $S_{q}$ is not null for $q<-N$.

This fact has been noted for $q>-(1+N)$ in [6, Lemma 3.8], and for shifts of a different kind in [35, Lemma 7].

We make a digression to note a consequence of the above computations.

Theorem 7.4. If $\left\{b_{k}\right\}$ is increasing, then $\left(S_{b_{j}}^{*}\right)^{m} \rightarrow 0$ as $m \rightarrow \infty$ in the strong operator topology. Further, $\left(S_{q_{j}}^{*}\right)^{m} \rightarrow 0$ as $m \rightarrow \infty$ in the strong operator topology if and only if $q \geq-N$.

Proof. As $m \rightarrow \infty,\left(S_{b_{j}}^{*}\right)^{m} \rightarrow 0$ in the strong operator topology if and only if $\left\|\left(S_{b_{j}}^{*}\right)^{m} f\right\|_{b}^{2}=\left[f, S_{b_{j}}^{m}\left(S_{b_{j}}^{*}\right)^{m} f\right]_{b} \rightarrow 0$ for every $f \in \mathcal{F}_{b}$. But

$$
\left[f, S_{b_{j}}^{m}\left(S_{b_{j}}^{*}\right)^{m} f\right]_{b}=\sum_{k=m}^{\infty} \sum_{|\alpha|=k} \frac{\left(1+\alpha_{j}-m\right)_{m}}{(1+k-m)_{m}} \frac{b_{k-m}}{b_{k}}\left|f_{\alpha}\right|^{2}\left\|z^{\alpha}\right\|_{b}^{2} .
$$


The first fraction is always $\leq 1$. If $\left\{b_{k}\right\}$ is increasing, then the second fraction is also $\leq 1$ and $\left[f, S_{b_{j}}^{m}\left(S_{b_{j}}^{*}\right)^{m} f\right]_{b} \rightarrow 0$ as $m \rightarrow \infty$ for all $f \in \mathcal{F}_{b}$ as in (33). The convergence for $q \geq-N$ also follows immediately.

For $q<-N$, the $\widetilde{f}$ above can be used again. For this function, $\alpha_{1}=k$ and the first fraction in $\left[\tilde{f}, S_{q_{1}}^{m}\left(S_{q_{1}}^{*}\right)^{m} \widetilde{f}\right]_{q}$ equals 1 . Then the only difference of $\left[\widetilde{f}, S_{q_{1}}^{m}\left(S_{q_{1}}^{*}\right)^{m} \widetilde{f}\right]_{q}$ from $\left\|J_{S_{q}}^{m}(I) \widetilde{f}\right\|_{q}^{2}$ in $(34)$ is that the power on $m$ now is $-(N+q)$. After estimating the norm of the tail end of $\tilde{f}$, we obtain $\left[\tilde{f}, S_{q_{1}}^{m}\left(S_{q_{1}}^{*}\right)^{m} \tilde{f}\right]_{q} \sim 1$, which does not tend to 0 as $m \rightarrow \infty$. For $j \neq 1$, just replace $z_{1}$ by $z_{j}$ in $\tilde{f}$.

Back to our main route, we next transform row contractions to any $\mathcal{F}_{b}$ building on the treatment in [17, Theorem 6.1.4].

Theorem 7.5. Let $T=\left(T_{1}, \ldots, T_{N}\right)$ be a row contraction on a Hilbert space $H$. For any $b=\left\{b_{k}\right\}$, there exists a unique bounded operator $L_{b}: \mathcal{F}_{b} \otimes \overline{\Delta_{T} H} \rightarrow H$ which is a contraction satisfying

$$
L_{b}\left(z^{\alpha} \otimes v\right)=\frac{1}{\sqrt{b_{|\alpha|}}} T^{\alpha} \Delta_{T} v \quad\left(v \in H, \alpha \in \mathbb{N}^{N}\right) .
$$

In particular, $L_{b}(1 \otimes v)=\Delta_{T} v$ for $v \in H$. If $T$ is null, then $L_{b}$ is a coisometry, that is, $L_{b} L_{b}^{*}=I_{H}$.

Proof. Uniqueness follows from the fact that members of $\mathcal{F}_{b}$ are holomorphic functions on $\mathbb{B}$ with Taylor expansions in terms of $\left\{z^{\alpha}\right\}$.

Set $H_{0}=\overline{\Delta_{T} H}$ and note that $H_{0}$ is a subspace of $H$. For each multi-index $\alpha$, put $k=|\alpha|$, and for $u \in H$, let

$$
y_{b_{\alpha}}=\frac{k !}{\alpha !} \sqrt{b_{k}} z^{\alpha} \otimes \Delta_{T} T^{* \alpha} u \quad \text { and } \quad y_{b_{k}}=\sum_{|\alpha|=k} y_{b_{\alpha}},
$$

which evidently belong to $E^{k} \otimes H_{0}$. In particular, $y_{b_{0}}=1 \otimes \Delta_{T} u$. By $(15)$ and (13), we have

$$
\begin{aligned}
\left\|y_{b_{k}}\right\|_{E^{k} \otimes H_{0}}^{2} & =\sum_{|\alpha|=k} \frac{(k !)^{2}}{(\alpha !)^{2}} b_{k}\left\|z^{\alpha}\right\|_{k}^{2}\left\|\Delta_{T} T^{* \alpha} u\right\|_{H}^{2}=\sum_{|\alpha|=k} \frac{k !}{\alpha !}\left[\Delta_{T} T^{* \alpha} u, \Delta_{T} T^{* \alpha} u\right]_{H} \\
& =\sum_{|\alpha|=k} \frac{k !}{\alpha !}\left[T^{\alpha} \Delta_{T}^{2} T^{* \alpha} u, u\right]_{H}=\left[J_{T}^{k}\left(\Delta_{T}^{2}\right) u, u\right]_{H} \\
& =\left[J_{T}^{k}\left(I-J_{T}(I)\right) u, u\right]_{H}=\left[\left(J_{T}^{k}(I)-J_{T}^{k+1}(I)\right) u, u\right]_{H} .
\end{aligned}
$$

Define an operator $A_{b}: H \rightarrow \mathcal{F}_{b} \otimes H_{0}$ by $A_{b} u=\left(y_{b_{0}}, y_{b_{1}}, y_{b_{2}}, \ldots\right)$. Then

$$
\begin{aligned}
\left\|A_{b} u\right\|_{\mathcal{F}_{b} \otimes H_{0}}^{2} & =\sum_{k=0}^{\infty}\left\|y_{b_{k}}\right\|_{E^{k} \otimes H_{0}}^{2}=\sum_{k=0}^{\infty}\left[\left(J_{T}^{k}(I)-J_{T}^{k+1}(I)\right) u, u\right]_{H} \\
& =\|u\|_{H}^{2}-\left[T_{\infty} u, u\right]_{H} \leq\|u\|_{H}^{2} \quad(u \in H) .
\end{aligned}
$$

Thus $\left\|A_{b}\right\| \leq 1$. Equality holds and $A_{b}$ is an isometry if $T$ is null. 
We now define $L_{b}=A_{b}^{*}$. Then $\left\|L_{b}\right\| \leq 1$, and $L_{b}$ is a coisometry if $T$ is null. Using (15) and (13), for $u \in H, v \in H_{0}$, and a multi-index $\alpha$, we compute

$$
\begin{aligned}
{\left[L_{b}\left(z^{\alpha} \otimes v\right), u\right]_{H} } & =\left[z^{\alpha} \otimes v, A_{b} u\right]_{\mathcal{F}_{b} \otimes H_{0}}=\left[z^{\alpha} \otimes v, y_{b_{\alpha}}\right]_{E^{k} \otimes H_{0}} \\
& =\left[z^{\alpha} \otimes v, \frac{k !}{\alpha !} \sqrt{b_{k}} z^{\alpha} \otimes \Delta_{T} T^{* \alpha} u\right]_{E^{k} \otimes H_{0}} \\
& =\frac{k !}{\alpha !} \sqrt{b_{k}}\left\|z^{\alpha}\right\|_{k}^{2}\left[v, \Delta_{T} T^{* \alpha} u\right]_{H}=\frac{1}{\sqrt{b_{k}}}\left[T^{\alpha} \Delta_{T} v, u\right]_{H},
\end{aligned}
$$

which establishes (35).

This theorem naturally leads to von Neumann inequalities, one with respect to each $\mathcal{F}_{b}$, which are new even when $N=1$.

Theorem 7.6. Let $T=\left(T_{1}, \ldots, T_{N}\right)$ be a row contraction on a Hilbert space $H$. Let $b$ be a weight sequence chosen with the property that $\left\{\frac{b_{k+m}}{b_{m}}\right\}$ is bounded above by a constant $K_{k}>0$ that depends only on $k$, and let $p(z)=\sum_{k=0}^{d} p_{k}(z)$ be a polynomial in $N$ complex variables, where $p_{k}$ is homogeneous of degree $k$. Then

$$
\|p(T)\| \leq \sum_{k=0}^{d} \sqrt{K_{k}}\left\|p_{k}\left(S_{b}\right)\right\|
$$

where $S_{b}$ is the b-shift.

Proof. By Theorem 7.5, we have a contraction $L_{b}: \mathcal{F}_{b} \otimes H_{0} \rightarrow H$ satisfying (35); equivalently,

$$
L_{b}\left(S_{b}^{\alpha} \otimes I_{H_{0}}\right)(1 \otimes v)=\frac{1}{\sqrt{b_{k}}} T^{\alpha} \Delta_{T} v \quad\left(v \in H_{0}, \alpha \in \mathbb{N}^{N}\right),
$$

where we continue to use $H_{0}=\overline{\Delta_{T} H}$ and $k=|\alpha|$. Let $\beta$ be another multi-index and $m=|\beta|$. Then

$$
L_{b}\left(S_{b}^{\alpha} \otimes I_{H_{0}}\right)\left(z^{\beta} \otimes v\right)=L_{b}\left(z^{\alpha+\beta} \otimes v\right)=\frac{1}{\sqrt{b_{k+m}}} T^{\alpha} \Delta_{T} v=\sqrt{\frac{b_{m}}{b_{k+m}}} T^{\alpha} L_{b}\left(z^{\beta} \otimes v\right)
$$

and

$$
L_{b}\left(S_{b}^{\alpha} \otimes I_{H_{0}}\right)\left(g_{k} \otimes v\right)=T^{\alpha} L_{b}(f \otimes v),
$$

where

$$
f(z)=\sum_{\beta} f_{\beta} z^{\beta} \in \mathcal{F}_{b}, \quad g_{k}(z)=\sum_{\beta} \sqrt{\frac{b_{k+m}}{b_{m}}} f_{\beta} z^{\beta},
$$

and $g_{k} \in \mathcal{F}_{b}$ by the assumption on $b$ since $\left\|g_{k}\right\|_{\mathcal{F}_{b}} \leq \sqrt{K_{k}}\|f\|_{\mathcal{F}_{b}}$.

We first consider the case $T$ is null under which $L_{b}^{*}$ is an isometry. Given an arbitrary $u \in H$, let $f \otimes v=L_{b}^{*} u$. Then $\|u\|_{H}=\|f \otimes v\|_{\mathcal{F}_{b} \otimes H}=\|f\|_{\mathcal{F}_{b}}\|v\|_{H}$. 
It follows that $T^{\alpha} L_{b}(f \otimes v)=T^{\alpha} L_{b} L_{b}^{*} u=T^{\alpha} u=L_{b}\left(S_{b}^{\alpha} \otimes I_{H_{0}}\right)\left(g_{k} \otimes v\right)$ and $p(T) u=L_{b} \sum_{k=0}^{d} p_{k}\left(S_{b}\right)\left(g_{k}\right) \otimes v$. Thus

$$
\begin{aligned}
\|p(T) u\|_{H} & =\sum_{k=0}^{d}\left\|p_{k}\left(S_{b}\right) g_{k}\right\|_{\mathcal{F}_{b}}\|v\|_{H} \leq \sum_{k=0}^{d}\left\|p_{k}\left(S_{b}\right)\right\|\left\|g_{k}\right\|_{\mathcal{F}_{b}}\|v\|_{H} \\
& \leq \sum_{k=0}^{d} \sqrt{K_{k}}\left\|p_{k}\left(S_{b}\right)\right\|\|f\|_{\mathcal{F}_{b}}\|v\|_{H}=\sum_{k=0}^{d} \sqrt{K_{k}}\left\|p_{k}\left(S_{b}\right)\right\|\|u\|_{H}
\end{aligned}
$$

and $\|p(T)\| \leq \sum_{k=0}^{d} \sqrt{K_{k}}\left\|p_{k}\left(S_{b}\right)\right\|$ when $T$ is null.

If $T$ is not null, for $0<r<1$, set $r T=\left(r T_{1}, \ldots, r T_{N}\right)$, which is null since $(r T)(r T)^{*} \leq r^{2} I$. Applying the above to $r T$, we obtain the same upper bound on $\|p(r T)\|$ which is independent of $r$ or $T$. Letting $r \rightarrow 1^{-}$finishes the proof.

When we have $\mathcal{F}_{b}=\mathcal{A}$, the Drury-Arveson space, and only then, we have $g_{k}=f$ for all $k$ in the above proof. Then (36) yields $T^{\alpha}=L_{b}\left(S_{b}^{\alpha} \otimes I_{H_{0}}\right) L_{b}^{*}$ when $T$ is null. From here the von Neumann inequality of [23, Theorem; 9, Theorem 8.1] is obtained, which is $\|p(T)\| \leq\left\|p\left(S_{-N}\right)\right\|$. Clearly, the inequality of this particular case is in general sharper than those of the other spaces considered in this work.

The assumption on $T$ in Theorem 7.6 is that $I-T T^{*} \geq 0$. In [37, Corollary 5.4], this condition is replaced by the positivity of more general polynomial expressions in $T$ and $T^{*}$, which also involves a consideration of Bergman spaces on domains determined by a similar polynomial inequality.

Remark 7.7. Let us see that the condition on $b$ in Theorem 7.6 is satisfied by the weight sequences $b_{q}$ of all the Dirichlet spaces $\mathcal{F}_{q}$. It is enough to check those other than the Drury-Arveson space $F_{-N}$. If $q>-(1+N)$, then

$$
\frac{b_{k+m}(q)}{b_{m}(q)}=\frac{(1+N+q+m)_{k}}{(1+m)_{k}} \text { and } \lim _{m \rightarrow \infty} \frac{b_{k+m}(q)}{b_{m}(q)}=1 .
$$

In the subcase $q>-N$, then the ratios are all $>1$ and the smallest

$$
K_{k}=\frac{b_{k}(q)}{b_{0}(q)}=b_{k}(q)=\frac{(1+N+q)_{k}}{k !}>1 .
$$

In the other subcase $-(1+N)<q<-N$, then the ratios are all $<1$ and the smallest

$$
K_{k}=\lim _{m \rightarrow \infty} \frac{b_{k+m}(q)}{b_{m}(q)}=1
$$

If $q \leq-(1+N)$, then

$$
\frac{b_{k+m}(q)}{b_{m}(q)}=\frac{(1+m)_{k}}{(1-(N+q)+m)_{k}} \text { and } \lim _{m \rightarrow \infty} \frac{b_{k+m}(q)}{b_{m}(q)}=1 .
$$

Since the ratios are all less than 1 now, the smallest $K_{k}=1$ again. 


\section{Spectra and Commutants}

We continue our investigation of the shift operators on the $\mathcal{F}_{b}$. We observe that they are weighted shift operators when (and only when) $N=1$. This yields a multitude of properties for them. We touch upon some that are related to their spectra. Later we determine the commutants of the shifts independently of $N$; they turn out to be the multiplier algebras of the $\mathcal{F}_{b}$ as can be expected.

All our shifts are undoubtedly one-to-one. By Proposition 3.4,

$$
S_{b_{j}} z^{\alpha}=\sqrt{\frac{\alpha !}{b_{|\alpha|}|\alpha| !}} S_{b_{j}} O_{\alpha}^{b}(z)=z^{\alpha+e_{j}}=\sqrt{\frac{\alpha !\left(1+\alpha_{j}\right)}{b_{1+|\alpha|}(1+|\alpha|) !}} O_{\alpha+e_{j}}^{b}(z),
$$

so

$$
S_{b_{j}} O_{\alpha}^{b}(z)=\sqrt{\frac{b_{|\alpha|}\left(1+\alpha_{j}\right)}{b_{1+|\alpha|}(1+|\alpha|)}} O_{\alpha+e_{j}}^{b}(z) .
$$

Proposition 8.1. When $N=1$, the shift $S_{b}$ is a weighted shift operator with the weight sequence

$$
\omega_{b}(k)=\sqrt{\frac{b_{k}}{b_{1+k}}}=\frac{\left\|z^{1+k}\right\|_{b}}{\left\|z^{k}\right\|_{b}} \quad(k=0,1,2, \ldots) .
$$

For $S_{q}$, the weight sequence has the form

$$
\omega_{q}(k)= \begin{cases}\sqrt{\frac{1+k}{2+q+k}} & \text { if } q>-2, \\ \sqrt{\frac{-q+k}{1+k}} & \text { if } q \leq-2 .\end{cases}
$$

The weight sequence of the weighted shift $S_{b}$ is not the same as the weight sequence $\left\{b_{k}\right\}$ of the weighted Fock space $\mathcal{F}_{b}$, although they are closely related by this proposition. When $N>1$, it is clear that the shifts $S_{b_{j}}$ and $S_{q_{j}}$ are not weighted shifts.

Every shift is a multiplication operator. Thus by (23), if $w \in \mathbb{B}$, then $\bar{w}_{j}$ is an eigenvalue of $S_{b_{j}}$ with common eigenvector $K_{b}(\cdot, w)$ for $j=1, \ldots, N$, that is,

$$
S_{b_{j}}^{*} K_{b}(\cdot, w)=\bar{w}_{j} K_{b}(\cdot, w) .
$$

In particular, 0 is an eigenvalue of each $S_{b_{j}}^{*}$ with eigenvector 1 . If $N=1$, we prove that points of $\mathbb{D}$ are the only eigenvalues of $S_{b}^{*}$ under a mild condition.

Theorem 8.2. Let $N=1$ and recall that $Z_{b}$ is defined in (3).

(i) We have $\sigma_{p}\left(S_{b}\right)=\emptyset$ and $\sqrt{Z_{b}} \mathbb{D} \subset \sigma_{p}\left(S_{b}^{*}\right) \subset \sqrt{Z_{b}} \overline{\mathbb{D}}$. In addition, all eigenspaces of $S_{b}^{*}$ are one-dimensional.

(ii) If also $\left\{1 / \omega_{b}(k)\right\}$ is bounded, then $\operatorname{im}\left(S_{b}\right)=\left\{f \in \mathcal{F}_{b}: f(0)=0\right\}$. 
(iii) If further $\lim _{k \rightarrow \infty} \omega_{b}(k)$ exists, then it is equal to $\sqrt{Z_{b}} \geq 1$, and we have $\sigma\left(S_{b}\right)=\sqrt{Z_{b}} \overline{\mathbb{D}}, \sigma_{p}\left(S_{b}^{*}\right)=\sqrt{Z_{b}} \mathbb{D}, \sigma_{e}\left(S_{b}\right)=\sqrt{Z_{b}} \partial \mathbb{D}$.

(iv) With $S_{q}, \lim _{k \rightarrow \infty} \omega_{q}(k)=\sqrt{Z_{q}}=1$ always exists and $\left\{1 / \omega_{q}(k)\right\}$ is thus bounded.

Proof. (i) In [42, p. 70], the quantity $r_{2}$ is defined. In our case, it is

$$
r_{2}=\liminf _{k \rightarrow \infty}\left(\omega_{b}(0) \cdots \omega_{b}(k-1)\right)^{1 / k}=\sqrt{\liminf _{k \rightarrow \infty} \frac{1}{b_{k}^{1 / k}}}=\frac{1}{\sqrt{\limsup _{k \rightarrow \infty} b_{k}^{1 / k}}}=\sqrt{Z_{b}}
$$

using Proposition 8.1. The claims now follow from [42, Theorem 8].

(ii) Without any condition on weight sequences, clearly $\operatorname{im}\left(S_{b}\right)$ is a subset of $\left\{f \in \mathcal{F}_{b}: f(0)=0\right\}$ which is closed since point evaluations are continuous on $\mathcal{F}_{b}$ by Theorem 3.2. Conversely, if $f(z)=\sum_{k=1}^{\infty} f_{k} z^{k} \in\left\{f \in \mathcal{F}_{b}: f(0)=0\right\}$, then $f(z)=z g(z)$ for some $g \in H(\mathbb{D})$. But

$$
\|g\|_{\mathcal{F}_{b}}=\sum_{k=1}^{\infty} \frac{\left|f_{k}\right|^{2}}{b_{k-1}}=\sum_{k=1}^{\infty} \frac{\left|f_{k}\right|^{2}}{b_{k}} \frac{b_{k}}{b_{k-1}}<\infty
$$

by the condition on $\left\{1 / \omega_{b}(k)\right\}$. This shows that $g \in \mathcal{F}_{b}$ and $f \in \operatorname{im}\left(S_{b}\right)$.

(iii) We have

$$
\lim _{k \rightarrow \infty} \omega_{b}(k)=\sqrt{\lim _{k \rightarrow \infty} \frac{b_{k}}{b_{1+k}}}=\sqrt{\frac{1}{\lim _{k \rightarrow \infty} b_{k}^{1 / k}}}=\sqrt{Z_{b}} \geq 1
$$

by [40, Theorem 3.37] and Proposition 8.1. Then the claim about $\sigma\left(S_{b}\right)$ follows from [42, Proposition 15].

Next we show that $\sqrt{Z_{b}} e^{i \theta}$ is not an eigenvalue of $S_{b}^{*}$. If it were, we would have an associated eigenvector $f(z)=\sum_{k=0}^{\infty} f_{k} z^{k} \in \mathcal{F}_{b}$. Writing down what this means in detail and solving for $f_{k}$ would give $f_{k}=\sqrt{Z_{b}} e^{i k \theta} b_{k} f_{0}$. We need $\sum_{k=0}^{\infty} Z_{b}^{k} b_{k}<\infty$ by (14) since $f \in \mathcal{F}_{b}$. But $Z_{b}^{k} \sim b_{k}^{-1}$ by (38) and such an $f$ would never be in $\mathcal{F}_{b}$. This proves the claim about $\sigma_{p}\left(S_{b}^{*}\right)$.

Let $\widetilde{S}$ be the weighted shift on $\mathcal{F}_{b}$ with the constant weight sequence $\{1,1, \ldots\}$. Then $S_{b}-\sqrt{Z_{b}} \widetilde{S}$ is a weighted shift whose weight sequence converges to 0 , which implies that it is compact by [42, Proposition 4]. So $S_{b}$ and $\sqrt{Z_{b}} \widetilde{S}$ are compact perturbations of each other and have the same essential spectrum. But $\widetilde{S}$ acts just like the unilateral shift on the Hardy space $H^{2}$ and thus $\sigma(\widetilde{S})=\partial \mathbb{D}$. This proves the claim about $\sigma_{e}\left(S_{b}\right)$.

(iv) That the limit of the weight sequence is 1 is clear from Proposition 8.1. Then we have $\sigma\left(S_{q}\right)=\overline{\mathbb{D}}, \sigma_{p}\left(S_{q}\right)=\emptyset, \sigma_{p}\left(S_{q}^{*}\right)=\mathbb{D}$, and $\sigma_{e}\left(S_{q}\right)=\partial \mathbb{D}$.

We turn to a slightly different topic now. When $N=1$, the commutant $\left\{S_{b}\right\}^{\prime}$ is the multiplier algebra $\mathcal{M}\left(\mathcal{F}_{b}\right)$ by $[42$, Theorem $3(\mathrm{~b})]$, because $S_{b}$ is a weighted shift. The starting point of such results is [41, Theorem 1], which deals with $\left\{S_{q}\right\}^{\prime}$ 
with $q=-1$. In fact, a little more generally, $\left\{S_{q}\right\}^{\prime}=H^{\infty}$ for all $q \geq-1$ with equal norms by the remarks early in Sec. 5 . The more interesting question is what would the commutant $\left\{S_{b_{1}}, \ldots, S_{b_{N}}\right\}^{\prime}$ be when $N>1$ since none of the operators $S_{b_{j}}$ is a weighted shift. It is clear that if $g \in \mathcal{M}\left(\mathcal{F}_{b}\right)$, then $M_{g}$ commutes with $S_{b_{1}}, \ldots, S_{b_{N}}$ since they are all multiplication operators, that is, $\mathcal{M}\left(\mathcal{F}_{b}\right) \subset\left\{S_{b_{1}}, \ldots, S_{b_{N}}\right\}^{\prime}$. Again there are no others although the proof is more involved.

Lemma 8.3. Let $\lambda=\left(\lambda_{1}, \ldots, \lambda_{N}\right) \in \mathbb{B}$. If $f \in \mathcal{F}_{b}$ is a common eigenvector of $S_{b_{1}}^{*}, \ldots, S_{b_{N}}^{*}$ associated to the eigenvalues $\lambda_{1}, \ldots, \lambda_{N}$, respectively, then $f$ is unique and $f(z)=f_{(0, \ldots, 0)} K_{b}(z, \bar{\lambda})$. Equivalently, $\operatorname{dim} \bigcap_{j=1}^{N} \operatorname{ker}\left(S_{b_{j}}^{*}-\lambda_{j} I\right)=1$.

Proof. By (24), the hypothesis is for $j=1, \ldots, N$ and $z \in \mathbb{B}$ that

$$
S_{b_{j}}^{*} f(z)=\sum_{|\alpha|>0} f_{\alpha} \frac{\alpha_{j}}{|\alpha|} \frac{b_{|\alpha|-1}}{b_{|\alpha|}} z^{\alpha-e_{j}}=\sum_{\beta} f_{\beta+e_{j}} \frac{1+\beta_{j}}{1+|\beta|} \frac{b_{|\beta|}}{b_{1+|\beta|}} z^{\beta}=\sum_{\beta} \lambda_{j} f_{\beta} z^{\beta} .
$$

Equating the coefficients of $z^{\beta}$ on the two sides and reverting to $\alpha$ yields

$$
f_{\alpha}=\lambda_{j} \frac{|\alpha|}{\alpha_{j}} \frac{b_{|\alpha|}}{b_{|\alpha|-1}} f_{\alpha-e_{j}} \quad\left(j=1, \ldots, N, \alpha_{j} \neq 0\right) .
$$

We can reach any $f_{\alpha}$ from $f_{(0, \ldots, 0)}$, because if $\alpha_{j} \neq 0$, we use the $j$ th formula repeatedly. This allows us to construct $f$ by determining all the $f_{\alpha}$ in terms of $f_{(0, \ldots, 0)}$. But a particular $f_{\alpha}$ can be obtained using the above formulas in different orders. These applications do not give a different $f_{\alpha}$ from each other, because applying the $j$ th formula is like applying $S_{b_{j}}$, and we know the $S_{b_{j}}$ commute with each other. Thus $f$ is unique up to a multiplicative constant $f_{(0, \ldots, 0)}$.

So we take one path from $(0, \ldots, 0)$ to $\alpha=\left(\alpha_{1}, \ldots, \alpha_{N}\right)$ and compute

$$
\begin{aligned}
f_{\alpha} & =\lambda_{1} \frac{|\alpha|}{\alpha_{1}} \frac{b_{|\alpha|}}{b_{|\alpha|-1}} f_{\alpha-e_{1}}=\lambda_{1}^{2} \frac{|\alpha|(|\alpha|-1)}{\alpha_{1}\left(\alpha_{1}-1\right)} \frac{b_{|\alpha|}}{b_{|\alpha|-1}} \frac{b_{|\alpha|-1}}{b_{|\alpha|-2}} f_{\alpha-2 e_{1}}=\cdots \\
& =\lambda_{1}^{\alpha_{1}} \frac{|\alpha|(|\alpha|-1) \cdots\left(|\alpha|-\alpha_{1}+1\right)}{\alpha_{1} !} \frac{b_{|\alpha|}}{b_{|\alpha|-\alpha_{1}}} f_{\alpha-\alpha_{1} e_{1}}=\cdots \\
& =\lambda_{1}^{\alpha_{1}} \lambda_{2}^{\alpha_{2}} \frac{|\alpha|(|\alpha|-1) \cdots\left(|\alpha|-\alpha_{1}-\alpha_{2}+1\right)}{\alpha_{1} ! \alpha_{2} !} \frac{b_{|\alpha|}}{b_{|\alpha|-\alpha_{1}-\alpha_{2}}} f_{\alpha-\alpha_{1} e_{1}-\alpha_{2} e_{2}}=\cdots \\
& =\lambda_{1}^{\alpha_{1}} \lambda_{2}^{\alpha_{2}} \cdots \lambda_{N}^{\alpha_{N}} \frac{|\alpha| !}{\alpha_{1} ! \alpha_{2} ! \cdots \alpha_{N} !} \frac{b_{|\alpha|}}{b_{0}} f_{(0, \ldots, 0)}=\lambda^{\alpha} \frac{|\alpha| !}{\alpha !} b_{|\alpha|} f_{(0, \ldots, 0)} .
\end{aligned}
$$

Therefore

$$
\begin{aligned}
f(z) & =\sum_{\alpha} f_{\alpha} z^{\alpha}=\sum_{\alpha} \lambda^{\alpha} \frac{|\alpha| !}{\alpha !} b_{|\alpha|} f_{(0, \ldots, 0)} z^{\alpha}=f_{(0, \ldots, 0)} \sum_{k=0}^{\infty} b_{k} \sum_{|\alpha|=k} \frac{k !}{\alpha !} \lambda^{\alpha} z^{\alpha} \\
& =f_{(0, \ldots, 0)} \sum_{k=0}^{\infty} b_{k}\langle z, \bar{\lambda}\rangle^{k}=f_{(0, \ldots, 0)} K_{b}(z, \bar{\lambda}) \quad(z \in \mathbb{B})
\end{aligned}
$$

by $(7)$. 
Pieces of the proofs of the remaining two theorems in this section are rather standard. But it is not possible to find in the literature what we need in full, so we provide the details for completeness. We abbreviate $\left\{S_{b_{1}}, \ldots, S_{b_{N}}\right\}$ to $\left\{S_{b}\right\}$.

Theorem 8.4. The commutant $\left\{S_{b}\right\}^{\prime}$ equals $\mathcal{M}\left(\mathcal{F}_{b}\right)$.

Proof. The proof rests on the fact that the intersection of the eigenspaces of the $S_{b_{j}}^{*}$ is one-dimensional. Suppose $T \in\left\{S_{b}\right\}^{\prime}$ so that $T S_{b_{j}}=S_{b_{j}} T$ for $j=1, \ldots, N$. Then $T^{*} S_{b_{j}}^{*}=S_{b_{j}}^{*} T^{*}$ as well. This shows that $T^{*} 1$ is a constant since $S_{b_{j}}^{*} 1=0$. Let $w=\left(w_{1}, \ldots, w_{N}\right) \in \mathbb{B}$. By $(37)$,

$$
T^{*} K_{b}(\cdot, w)=\frac{1}{\bar{w}_{j}} T^{*} S_{b_{j}}^{*} K_{b}(\cdot, w)=\frac{1}{\bar{w}_{j}} S_{b_{j}}^{*} T^{*} K_{b}(\cdot, w)
$$

for $w_{j} \neq 0$. Then $S_{b_{j}}^{*}\left(T^{*} K_{b}(\cdot, w)\right)=\bar{w}_{j}\left(T^{*} K_{b}(\cdot, w)\right)$, which is true if $w_{j}=0$ also since then the series expansion of $K_{b}(\cdot, w)$ contains no $z_{j}$. So $T^{*} K_{b}(\cdot, w)$ is a common eigenvector of the $S_{b_{j}}^{*}$ with the eigenvalues $\bar{w}_{j}$. Lemma 8.3 implies the existence of a constant $\overline{g(w)}$ such that $T^{*} K_{b}(z, w)=\overline{g(w)} K_{b}(z, w)$. This means that $\overline{g(w)} \in \sigma\left(T^{*}\right)$, hence $g(w) \in \sigma(T)$, and thus $|g(w)| \leq\|T\|$; that is, $g$ is a bounded function of $w$. Next, the computation

$$
\begin{aligned}
g(w) & =g(w)\left[1, K_{b}(\cdot, w)\right]_{b}=\left[1, \overline{g(w)} K_{b}(\cdot, w)\right]_{b} \\
& =\left[1, T^{*} K_{b}(\cdot, w)\right]_{b}=\left[T 1, K_{b}(\cdot, w)\right]_{b}=T 1(w)
\end{aligned}
$$

yields that $g=T 1 \in \mathcal{F}_{b}$. Finally, for $f \in \mathcal{F}_{b}$, the reverse computation

$$
\begin{aligned}
T f(w) & =\left[T f, K_{b}(\cdot, w)\right]_{b}=\left[f, T^{*} K_{b}(\cdot, w)\right]_{b} \\
& =\left[f, \overline{g(w)} K_{b}(\cdot, w)\right]_{b}=g(w)\left[f, K_{b}(\cdot, w)\right]_{b}=g(w) f(w)
\end{aligned}
$$

shows that $T=M_{g} \in \mathcal{M}\left(\mathcal{F}_{b}\right)$.

Let $\mathcal{S}\left(S_{b}\right)$ be the smallest sot-closed algebra in $\mathcal{B}\left(\mathcal{F}_{b}\right)$ containing $\left\{S_{b}\right\}$ and $I$, $\mathcal{W}\left(S_{b}\right)$ be the smallest wot-closed algebra in $\mathcal{B}\left(\mathcal{F}_{b}\right)$ containing $\left\{S_{b}\right\}$ and $I$, and $\mathcal{W}^{*}\left(S_{b}\right)$ be the smallest weak ${ }^{*}$-closed algebra in $\mathcal{B}\left(\mathcal{F}_{b}\right)$ containing $\left\{S_{b}\right\}$ and $I$.

Theorem 8.5. We have $\mathcal{W}^{*}\left(S_{b}\right)=\mathcal{W}\left(S_{b}\right)=\mathcal{S}\left(S_{b}\right)=\left\{S_{b}\right\}^{\prime \prime}=\left\{S_{b}\right\}^{\prime}=\mathcal{M}\left(\mathcal{F}_{b}\right)$.

Proof. It is easy to see that a commutant is sot-closed, and thus it is also wotclosed since it is a subspace; see [33, Theorem 4.2.7]. Thus $\mathcal{M}\left(\mathcal{F}_{b}\right)$ is sot-closed and wot-closed by Theorem 8.4. The same result implies also $\mathcal{W}\left(S_{b}\right)=\mathcal{S}\left(S_{b}\right)$.

It is clear that $\left\{S_{b}\right\} \subset\left\{S_{b}\right\}^{\prime}$ since individual shifts commute with each other. Hence $\left\{S_{b}\right\} \subset\left\{S_{b}\right\}^{\prime \prime} \subset\left\{S_{b}\right\}^{\prime}$ as well. Also $\mathcal{W}\left(S_{b}\right) \subset\left\{S_{b}\right\}^{\prime \prime}$ by the remarks in the previous paragraph. Moreover, weak ${ }^{*}$-convergence implies wot-convergence; see $\left[33\right.$, p. 126]. Then $\mathcal{W}^{*}\left(S_{b}\right) \subset \mathcal{W}\left(S_{b}\right)$. 
Finally, let $M_{g} \in \mathcal{M}\left(\mathcal{F}_{b}\right)$. Then $g \in \mathcal{F}_{b} \cap H^{\infty}$ by the general remarks on multipliers in Sec. 5. By Definition 3.1 and Theorem 3.2, there is a sequence $\left\{p_{n}\right\}$ of polynomials converging to $g$ in $\|\cdot\|_{\mathcal{F}_{b}}$ and uniformly on compact subsets of $\mathbb{B}$. Then

$$
\begin{aligned}
{\left[p_{n}\left(S_{b}\right) f, K_{b}(\cdot, w)\right]_{b} } & =\left[p_{n} f, K_{b}(\cdot, w)\right]_{b}=p_{n}(w) f(w) \\
\rightarrow g(w) f(w) & =\left[g f, K_{b}(\cdot, w)\right]_{b}=\left[M_{g} f, K_{b}(\cdot, w)\right]_{b}
\end{aligned}
$$

for any $f \in \mathcal{F}_{b}$ and $w \in \mathbb{B}$. Thus $p_{n}\left(S_{b}\right) \rightarrow M_{g}$ in wot and $M_{g} \in \mathcal{W}\left(S_{b}\right)$. Since $\left\{p_{n}\left(S_{b}\right)\right\}$ is a sequence and $\mathcal{F}_{b}$ is separable by definition, also $p_{n}\left(S_{b}\right) \rightarrow M_{g}$ weak* and $\mathcal{M}\left(\mathcal{F}_{b}\right) \subset \mathcal{W}^{*}\left(S_{b}\right)$ by [20, Proposition 20.3(a)].

\section{Almost Normality}

We now present several results on the commutators, hyponormality, and subnormality of the shift operators on $\mathcal{F}_{b}$ and $\mathcal{F}_{q}$. Our unilateral shifts singly or as a tuple are never normal as are immediately seen by the results of Sec. 6. So we investigate the conditions under which they are hyponormal or essentially normal or subnormal.

Definition 9.1. We call a commuting $N$-tuple of operators $T=\left(T_{1}, \ldots, T_{N}\right)$ on a Hilbert space $H$ tuple-hyponormal if $T^{*} T-T T^{*} \geq 0$ using Notation 6.1 . We call $T$ tuple-essentially normal if $T^{*} T-T T^{*}$ is compact. We call $T$ tuple-subnormal if there is a Hilbert space $H^{\prime} \supset H$ and on $H^{\prime}$ a commuting $N$-tuple $T^{\prime}=\left(T_{1}^{\prime}, \ldots, T_{N}^{\prime}\right)$ of normal operators such that each $T_{j}$ is the restriction of $T_{j}^{\prime}$ to $H$.

When $N=1$, the concepts with the tuple prefix reduce to the usual concepts without it. The definition of tuple-subnormality is from [9, Remark 3.10].

Theorem 9.2. The operator $S_{b}$ is tuple-hyponormal if and only if the weight sequence satisfies $(N+k) b_{k}^{2} \geq(1+k) b_{k-1} b_{k+1}$ for $k=1,2, \ldots$ In particular, when $N=1, S_{b}$ is tuple-hyponormal if and only if $\left\{b_{k}\right\}$ is a log-concave sequence.

So for $N=1, S_{q}$ is (tuple-)hyponormal if and only if $q \geq-1$; for $N=2, S_{q}$ is tuple-hyponormal if and only if $q \geq-5 / 2$ or $-4 \leq q \leq-3$; for $N \geq 3, S_{q}$ is tuple-hyponormal if and only if $q \geq\left(1-N-N^{2}\right) / N$ or $q \leq-(1+N)$.

Proof. By (25),

$$
\left(S_{b}^{*} S_{b}-S_{b} S_{b}^{*}\right) f=\frac{N}{b_{1}} f_{0}+\sum_{k=1}^{\infty}\left(\frac{N+k}{1+k} \frac{b_{k}}{b_{k+1}}-\frac{b_{k-1}}{b_{k}}\right) f_{k}
$$

and $N / b_{1}>0$. The first statement follows from Theorem 3.5. The second statement follows from the definition of a concave sequence which is $b_{k} \geq\left(b_{k-1}+b_{k+1}\right) / 2$. 
Next, by (27) and (28),

$$
\begin{aligned}
& \left(S_{q}^{*} S_{q}-S_{q} S_{q}^{*}\right) f \\
& \quad=\left\{\begin{array}{r}
\frac{N}{1+N+q} f_{0}+\sum_{k=1}^{\infty}\left[\frac{N+k}{1+N+q+k}-\frac{k}{N+q+k}\right] f_{k} \\
\text { if } q>-(1+N), \\
(1-N-q) N f_{0}+\sum_{k=1}^{\infty}\left[\frac{(1-N-q+k)(N+k)}{(1+k)^{2}}+\frac{N+q-k}{k}\right] f_{k} \\
\text { if } q \leq-(1+N) .
\end{array}\right.
\end{aligned}
$$

The coefficient of $f_{0}$ is always $\geq 0$.

For $q>-(1+N)$, the coefficient of $f_{k}$ is $\geq 0$ for all $k=1,2, \ldots$ if and only if $q \geq\left(1-N-N^{2}\right) / N$, which also yields the inequalities $q \geq-1$ for $N=1$ and $q \geq-5 / 2$ for $N=2$.

For $q \leq-(1+N)$, the coefficient of $f_{k}$ with $k \geq 1$ is nonnegative if and only if $q(1-N k+2 k) \geq-N+\left(N^{2}-3 N+1\right) k+(1-N) k^{2}$. The coefficient of $q$ on the left shows that we need to look at the cases $N=1, N=2$, and $N \geq 3$ separately. When $N=1$, we get $q \geq-1$, but we have to have $q \leq-(1+N)=-2$; so there are no new $q$ values. When $N=2$, we get $q \geq-4$, but we have to have $q \leq-(1+N)=-3$; so the new $q$ values are $-4 \leq q \leq-3$. Finally, when $N \geq 3$, the inequality for $q$ gives an upper bound for $q$ after a check for all $k \geq 1$, but this upper bound is actually greater than $-(1+N)$; so in this case too tuple-hyponormality obtains for all $q \leq-(1+N)$.

The case $N=1$ is outlined in [42, Lemma, p. 83].

The commutators of the individual $S_{b_{j}}$ and their adjoints are written easily using the formulas in Sec. 6. However, unless more is known about the detailed values of $\left\{b_{k}\right\}$, these formulas do not yield any meaningful result. So for such commutators, we confine ourselves to the $S_{q_{j}}$. It turns out that these formulas are useful due to the powerful radial differential operators $D_{s}^{t}$.

Proposition 9.3. For $i, j=1, \ldots, N$, we have

$$
\begin{aligned}
S_{q_{i}}^{*} S_{q_{j}} & -S_{q_{j}} S_{q_{i}}^{*} \\
= & \begin{cases}\frac{1}{1+N+q} D_{1+q}^{-1}\left(\delta_{i j} I-S_{q_{j}} S_{q_{i}}^{*}\right) & \text { if } q>-(1+N) \\
(1-N-q) D_{-1+q}^{1}\left(D_{-N}^{-1}\right)^{2}\left(\delta_{i j} I-S_{q_{j}} S_{q_{i}}^{*}\right)+K_{j} & \text { if } q \leq-(1+N),\end{cases}
\end{aligned}
$$

where

$$
K_{j}\left(z^{\alpha}\right)=\alpha_{j}(N+q)^{2}\left(D_{-N}^{-1}\right)^{2}\left(\left(R^{\prime}\right)^{-1}\right)^{2}\left(z^{\alpha}\right),
$$

$\left(R^{\prime}\right)^{-1}$ is given in (32), and $\delta_{i j}$ is the Kronecker delta. 
Proof. We show the details only for $i=j$; those results for $i \neq j$ are similar and in fact simpler. Also it is enough to show the action of operators on $z^{\alpha}$ for $|\alpha|>0$.

For $q>-(1+N)$, using (26) and (19) and simplifying, we have

$$
\left(S_{q_{j}}^{*} S_{q_{j}}-S_{q_{j}} S_{q_{j}}^{*}\right) z^{\alpha}=\frac{N+q+|\alpha|-\alpha_{j}}{(1+N+q+|\alpha|)(N+q+|\alpha|)} z^{\alpha}
$$

and

$$
\frac{1}{1+N+q} D_{1+q}^{-1}\left(I-S_{q_{j}} S_{q_{j}}^{*}\right) z^{\alpha}=\frac{1}{1+N+q+|\alpha|}\left(1-\frac{\alpha_{j}}{N+q+|\alpha|}\right) z^{\alpha} .
$$

These two expressions are the same.

For $q \leq-(1+N)$, we have instead

$$
\left(S_{q_{j}}^{*} S_{q_{j}}-S_{q_{j}} S_{q_{j}}^{*}\right) z^{\alpha}=\left(\frac{1+\alpha_{j}}{(1+|\alpha|)^{2}}(1-N-q+|\alpha|)-\frac{\alpha_{j}}{|\alpha|^{2}}(-N-q|\alpha|)\right) z^{\alpha}
$$

and

$$
(1-N-q) D_{-1+q}^{1}\left(D_{-N}^{-1}\right)^{2}\left(I-S_{q_{j}} S_{q_{j}}^{*}\right) z^{\alpha}=\frac{(1-N-q+|\alpha|)\left(|\alpha|^{2}+\alpha_{j}(N+q-|\alpha|)\right)}{|\alpha|^{2}(1+|\alpha|)^{2}} z^{\alpha} .
$$

The term $K_{j}\left(z^{\alpha}\right)$ exactly accounts for the difference between the right-hand sides of the last two formulas.

The case $q=-N$ of Proposition 9.3 is in [9, Proposition 5.3]. The following corollary is in [6, Corollary 3.5] with a completely different proof; its $q=-N$ case is in $[9, \mathrm{p} .192]$.

Corollary 9.4. Each $S_{q_{j}}$ is hyponormal if and only if $q \geq-N$.

Proof. By Proposition 4.4, all the radial differential operators encountered in Proposition 9.3 are positive. Consequently $K_{j} \geq 0$ as well. So everything depends on the positivity of $I-S_{q_{j}} S_{q_{j}}^{*}$. For $\alpha_{j}>0$, we have

$$
\left(I-S_{q_{j}} S_{q_{j}}^{*}\right) z^{\alpha}= \begin{cases}\frac{N+q+|\alpha|-\alpha_{j}}{N+q+|\alpha|} z^{\alpha} & \text { if } q>-(1+N), \\ \frac{|\alpha|\left(1-\alpha_{j}\right)+(N+q) \alpha_{j}}{|\alpha|^{2}} z^{\alpha} & \text { if } q \leq-(1+N) .\end{cases}
$$

The coefficient of all the $z^{\alpha}$ are positive if and only if $q \geq-N$. If $\alpha_{j}=0$, then $I-S_{q_{j}} S_{q_{j}}^{*}=I$ anyway.

The formulas developed above tell us also which operators are essentially normal.

Proposition 9.5. Every $S_{q}$ is tuple-essentially normal and every $S_{q_{j}}$ is essentially normal. Further, the commutator $S_{q_{i}}^{*} S_{q_{j}}-S_{q_{j}} S_{q_{i}}^{*} \in \mathcal{C}^{p}\left(\mathcal{F}_{q}\right)$ if and only if $p>N$.

The case $q=-N$ of the last claim is in [9, Proposition 5.3]. This fact is also called $p$-essential normality and has recently been extended to more general situations; see, for example, [27]. Note again that the condition $p>N$ is indepenent of $q$. 
Proof of Proposition 9.5. Consider the formula for $\left(S_{q}^{*} S_{q}-S_{q} S_{q}^{*}\right) f$ obtained in the proof of Theorem 9.2. The coefficient of $f_{k}$ there is $\sim 1 / k$ and Theorem 3.5 gives the result about $S_{q}$.

Next consider the formula for $S_{q_{j}}^{*} S_{q_{j}}-S_{q_{j}} S_{q_{j}}^{*}$ obtained in Proposition 9.3. There $I-S_{q_{j}}^{*} S_{q_{j}}$ is bounded by the work of Sec. 6 . It is composed with a radial differential operator of total order -1 which is compact by Proposition 4.4. The only remaining operator $K_{j}$ is also a radial differential operator of total order -3 (the coefficient $\alpha_{j}$ in $K_{j}\left(z^{\alpha}\right)$ indicates a derivative of order 1$)$ and is also compact.

For the final claim, we first show that $A_{i j}=\delta_{i j} I-S_{q_{j}} S_{q_{i}}^{*}$ is not compact. Suppose it is. If $i=j$, using (26) and summing on $i=1, \ldots, N$, we see that

$$
\sum_{i=1}^{N}\left(I-S_{q_{i}} S_{q_{i}}^{*}\right) z^{\alpha}=\frac{N^{2}+N q+(N-1)|\alpha|}{N+q+|\alpha|} z^{\alpha}
$$

is also compact. If $i \neq j$, summing on $i, j=1, \ldots, N$, we see that

$$
\sum_{i, j=1}^{N}\left(A_{i j}^{*} A_{i j}\right) z^{\alpha}=\frac{|\alpha|^{2}}{(N+q+|\alpha|)^{2}} z^{\alpha}
$$

is also compact. But these diagonal operators are not compact by Theorem 3.5. Then the Schatten ideal membership of the commutator depends solely on the radial differential operator of total order -1 multiplying $A_{i j}$ in Proposition 9.3. We finish as for the last claim in Proposition 4.4 obtaining the same condition.

We turn to subnormality. If $q \geq-1$, then $\mathcal{F}_{q}$ is a subspace of the Lebesgue space $\mathcal{L}_{q}^{2}$ with the same norm by Remark 4.7 . Then $S_{q}$ has the normal extension $T=\left(T_{1}^{\prime}, \ldots, T_{N}^{\prime}\right)$, where $T_{j}^{\prime}=M_{z_{j}}$ with $\left(T_{j}^{\prime}\right)^{*}=M_{\bar{z}_{j}}$. Thus $S_{q}$ is tuple-subnormal for $q \geq-1$. Theorem 6.1 in [6] contains the result that there is not any other tuple-subnormal $S_{q}$ for $q>-(1+N)$; the case $q=-N$ is given in [9, p. 180]; see also [26, Theorem 3.2]. It turns out that there is not any other subnormal $S_{q}$ for $q \leq-(1+N)$. Our different proof depends on the following result which is important in its own right.

Theorem 9.6. Let $C_{b}$ be an upper bound for the sequence $\left\{b_{k}^{1 / k}\right\}$ which is finite by (3). Then the geometric joint spectral radius of $S_{b}$ is at most $1 / \sqrt{C_{b}}$ and hence the Taylor joint spectrum of $S_{b}$ lies in the closed ball of this radius.

See [21, Definition 2.1] for the Taylor joint spectrum $\sigma_{T}$. See [18, p. 253] for the geometric joint spectral radius $r_{G}$.

Proof of Theorem 9.6. We follow the proof of [25, Lemma 2.5], where a similar computation is done for $S_{q}$ with $q>-(1+N)$. In [18, Theorem 1], $r_{G}$ is shown to be independent of the particular joint spectrum, and a formula for it is given in [34]. Using it and Proposition 3.4, we deduce that if we can find positive constants $C_{\alpha}$ 
such that $\left\|S_{b}^{\alpha} z^{\beta}\right\|_{b} \leq C_{\alpha}\left\|z^{\beta}\right\|_{b}$ for all multi-indices $\beta$, then

$$
r_{G}\left(S_{b}\right) \leq \lim _{m \rightarrow \infty}\left(\sum_{|\alpha|=m} \frac{m !}{\alpha !} C_{\alpha}^{2}\right)^{1 / 2 m} .
$$

Note that $|\alpha+\beta|=|\alpha|+|\beta|$. Then

$$
\left\|S_{b}^{\alpha} z^{\beta}\right\|_{b}^{2}=\left\|z^{\alpha+\beta}\right\|_{b}^{2}=\frac{(\alpha+\beta) !}{b_{|\alpha|+|\beta|}(|\alpha|+|\beta|) !} \leq \frac{(\alpha+\beta) !}{\beta !} \frac{C_{b}^{-|\alpha|}}{(1+|\beta|)_{|\alpha|}}\left\|z^{\beta}\right\|_{b}^{2},
$$

and by (1) and (2),

$$
\begin{aligned}
\sum_{|\alpha|=m} \frac{m !}{\alpha !}\left\|S_{b}^{\alpha} z^{\beta}\right\|_{b}^{2} & =\frac{\left\|z^{\beta}\right\|_{b}^{2}}{C_{b}^{m}} \frac{(1)_{m}}{(1+|\beta|)_{m}} \sum_{|\alpha|=m} \frac{(\alpha+\beta) !}{\alpha ! \beta !} \\
& \leq C C_{b}^{-m} m^{-|\beta|}\left\|z^{\beta}\right\|_{b}^{2} \sum_{|\alpha|=m} \frac{\left(1+\beta_{1}\right)_{\alpha_{1}}}{(1)_{\alpha_{1}}} \cdots \frac{\left(1+\beta_{N}\right)_{\alpha_{N}}}{(1)_{\alpha_{N}}} \\
& \leq C C_{b}^{-m} m^{-|\beta|}\left\|z^{\beta}\right\|_{b}^{2} m^{|\beta|} m^{N-1}=C C_{b}^{-m} m^{N-1}\left\|z^{\beta}\right\|_{b}^{2} .
\end{aligned}
$$

Taking the $2 m$ th root of the coefficient and letting $m \rightarrow \infty$ yield the desired result for $S_{b}$.

Corollary 9.7. For any $q \in \mathbb{R}, r_{G}\left(S_{q}\right) \leq 1$ and $\sigma_{T}\left(S_{q}\right) \subset \overline{\mathbb{B}}$.

Proof. We have $C_{b(q)}=1$ for all $q \in \mathbb{R}$.

Through other means, in $\left[26\right.$, Theorem 4.5], it is shown that $\sigma_{T}\left(S_{q}\right)=\overline{\mathbb{B}}$. The same result also finds the Taylor joint essential spectrum of $S_{q}$, but we find it in a different way in Corollary 10.16 below.

Theorem 9.8. The operator $S_{q}$ is tuple-subnormal if and only if $q \geq-1$.

Proof. Only the only if part needs a proof. So let $q<-1$ and suppose $S_{q}$ is tuplesubnormal. We use [10, Theorem 5.2] which states that a tuple $T$ is tuple-subnormal with $\sigma_{T}(T) \subset \overline{\mathbb{B}}$ if and only if

$$
h_{n}(T):=\left(I-J_{T^{*}}(I)\right)^{n}=\sum_{k=0}^{n}(-1)^{k}\left(\begin{array}{l}
n \\
k
\end{array}\right) \sum_{|\alpha|=k}\left(T^{\alpha}\right)^{*} T^{\alpha} \geq 0
$$

for every $n=1,2, \ldots$ The Taylor joint spectrum condition is provided by Corollary 9.7. We only need $n=1$ to arrive at a contradiction. Then $h_{1}\left(S_{q}\right)=I-S_{q}^{*} S_{q}$ with Notation 6.1 , and this is not positive if $q<-1$ by (31).

Corollary 9.9. Among the $\mathcal{F}_{q}$, the only spaces in which the norm of $f \in \mathcal{F}_{q}$ can be described by integration of $|f|^{2}$ against a positive measure on $\mathbb{C}^{N}$ are those with $q \geq-1$. 
Proof. This is of course [12, Theorem 4.3], but our proof is very different and immediate by [26, Proposition 3.1], where the condition in the statement of the theorem is proved to be equivalent to tuple-subnormality of the shift operator.

The limited case $q>-(1+N)$ of the following is also in [6, Corollary 3.5]; we give a completely different proof for all $q \in \mathbb{R}$.

Proposition 9.10. Each $S_{q_{j}}$ is subnormal if and only if $q \geq-N$.

Proof. For $q<-N, S_{q_{j}}$ is not subnormal since it is not hyponormal by Corollary 9.4. For $q \geq-N$, we use [1, Theorem 3.1] which states that an operator $A$ is subnormal and $\|A\| \leq 1$ if and only if $A$ is an $N$-hypercontraction for every $n=1,2, \ldots$ meaning that

$$
h_{n}(A):=\left(I-J_{A^{*}}(I)\right)^{n}=\sum_{k=0}^{n}(-1)^{k}\left(\begin{array}{l}
n \\
k
\end{array}\right)\left(A^{*}\right)^{k} A^{k} \geq 0
$$

for every $n=1,2, \ldots$ For any $n$ and multi-index $\alpha$, we have

$$
\begin{aligned}
h_{n}\left(S_{q_{j}}\right) z^{\alpha}= & \frac{N+q+|\alpha|-\alpha_{j}}{1+N+q+|\alpha|}\left(1-\frac{1+\alpha_{j}}{2+N+q+|\alpha|}\left(2-\frac{2+\alpha_{j}}{3+N+q+|\alpha|}\right.\right. \\
& \left.\left.\cdot\left(3-\frac{3+\alpha_{j}}{4+N+q+|\alpha|} \cdots\left(n-1-\frac{n-1+\alpha_{j}}{n+N+q+|\alpha|}\right)\right)\right)\right) z^{\alpha} .
\end{aligned}
$$

It suffices to check the case $q=-N$. The largest possible number after the minus sign in the first parentheses is $1 / 2$ and the smallest number after the minus sign in the second parentheses is 0 . In such a case, the coefficient of $z^{\alpha}$ is 0 . In all other cases, this coefficient is $\geq 0$. Thus $h_{n}\left(S_{q_{j}}\right) \geq 0$ for any $n$ and $j$ for $q \geq-N$, and $S_{q_{j}}$ is subnormal.

Let us record here that the hyponormality of an operator or a tuple $T$ is deduced from the positivity of operators of the form $I-T T^{*}$ and its subnormality from the positivity of operators of the form $I-T^{*} T$.

\section{Toeplitz Algebras}

We would like to investigate the $C^{*}$-algebras generated by the shift operators on $\mathcal{F}_{q}$. They are also called Toeplitz algebras because of their close connection to Toeplitz operators on weighted Bergman and Hardy spaces, those $\mathcal{F}_{q}$ with $q \geq-1$. However, in the few publications dealing with $q<-1$, these algebras are defined only with shift operators. It is our aim now to put back the Toeplitz operators to the Toeplitz algebras. Our approach is classical, but brings out the properties of these algebras better.

For $q>-1$, the Dirichlet spaces $\mathcal{F}_{q}$ are the Bergman spaces $A_{q}^{2}$ which are closed subspaces of the Lebesgue classes $\mathcal{L}_{q}^{2}$. There are on them the usual Toeplitz operators $T_{q, \phi}: \mathcal{F}_{q} \rightarrow \mathcal{F}_{q}$ whose symbols are measurable functions $\phi$ on $\mathbb{B}$ defined 
by $T_{q, \phi}:=P_{q} M_{\phi} i$, where $i: \mathcal{F}_{q} \rightarrow \mathcal{L}_{q}^{2}$ is the inclusion, $M_{\phi}: \mathcal{L}_{q}^{2} \rightarrow \mathcal{L}_{q}^{2}$ denotes multiplication by $\phi$, and $P_{q}: \mathcal{L}_{q}^{2} \rightarrow \mathcal{F}_{q}$ is the orthogonal projection. For $\phi(z)=z_{j}$, we actually have $T_{q, z_{j}}=S_{q_{j}}, j=1, \ldots, N$.

For $q \leq-1, \mathcal{F}_{q}$ need not be a subspace of $\mathcal{L}_{q}^{2}$ and the idea of projecting directly onto a subspace fails. In [5, Sec. 4], a more general family of Toeplitz operators acting on all $\mathcal{F}_{q}$ has been developed. The construction below works on any $\mathcal{F}_{q}$ with $q \in \mathbb{R}$ and in fact yields often different Toeplitz operators even on the weighted Bergman spaces $\mathcal{F}_{q}$ for which $q>-1$.

Throughout this section, we use the integral norm $\||\cdot|\|_{q}$ and the associated inner product $\llbracket \cdot, \cdot \rrbracket_{q}$ on $\mathcal{F}_{q}$ introduced in Remark 4.7 , because they give exact formulas and simplify our work.

Given $q \in \mathbb{R}$, fix $s$ so that $-q+2 s=0$ and fix also $t=-q+s$; then $q+2 t=0$ and $s+t=0$. (Actually, $=0$ is used here for simplicity; $>-1$ works just as fine.) The generalized Toeplitz operator $U_{q, \phi}: \mathcal{F}_{q} \rightarrow \mathcal{F}_{q}$ is defined by

$$
U_{q, \phi}:=P_{s} M_{\phi} I_{s}^{t}=P_{q / 2} M_{\phi} I_{q / 2}^{-q / 2}
$$

where $I_{s}^{t}: \mathcal{F}_{q} \rightarrow \mathcal{L}_{q}^{2}$ is one of the imbeddings introduced in Theorem 4.6 and $P_{s}$ : $\mathcal{L}_{q}^{2} \rightarrow \mathcal{F}_{q}$ is a generalized Bergman projection that is proved to be bounded in [31, Theorem 1.2] for the selection of the parameters $s, t$. It must be noted that for $q \leq-1$, we cannot use $P_{q}$ in place of $P_{q / 2}$. This form of a Toeplitz operator follows the classical form except that the usual inclusion $i$ is replaced by a different imbedding $I_{s}^{t}$. In [5, Theorem 4.5], it is shown that

$$
U_{q, \phi}=\left(D_{s}^{t}\right)^{-1} T_{0, \phi} D_{s}^{t}=D_{0}^{q / 2} T_{0, \phi} D_{q / 2}^{-q / 2}
$$

where $T_{0, \phi}$ is the usual Toeplitz operator on the unweighted Bergman space $\mathcal{F}_{0}$. By Corollary 4.8, $D_{s}^{t}$ moves us up to the Bergman space $\mathcal{F}_{0}, T_{0, \phi}$ acts on this Bergman space, and then $\left(D_{s}^{t}\right)^{-1}$ moves us back to the Besov space $\mathcal{F}_{q}$. From now on, we use $T_{0, \phi}$ only with first parameter $q=0$. For $q=0$ and only for it, $U_{0, \phi}=T_{0, \phi}$ and $U_{0, z_{j}}=T_{0, z_{j}}=S_{0_{j}}$, because

$$
\begin{aligned}
U_{q, z_{j}} z^{\alpha}= & \left(D_{0}^{q / 2} S_{0_{j}} D_{q / 2}^{-q / 2}\right) z^{\alpha}=\frac{b_{|\alpha|+1}(q / 2)}{b_{|\alpha|+1}(0)} \frac{b_{|\alpha|}(0)}{b_{|\alpha|}(q / 2)} z^{\alpha+e_{j}} \\
= & \begin{cases}\frac{1+N+q / 2+|\alpha|}{1+N+|\alpha|} z^{\alpha+e_{j}} & \text { if } q / 2>-(1+N), \\
\frac{(1+|\alpha|)^{2}}{(1+N+|\alpha|)(1-(N+q / 2)+|\alpha|)} z^{\alpha+e_{j}} & \text { if } q / 2 \leq-(1+N),\end{cases}
\end{aligned}
$$

by (19) and Definition 4.1. Note that the shift operator in the middle acts on the unweighted Bergman space $\mathcal{F}_{0}$.

Let us compute the adjoints. First for $q>-1$,

$$
T_{q, \phi}^{*}=i^{*} M_{\phi}^{*} P_{q}^{*}=P_{q} M_{\bar{\phi}} i=T_{q, \bar{\phi}} \quad \text { and } \quad T_{q, z_{j}}^{*}=T_{q, \bar{z}_{j}}=S_{q_{j}}^{*}
$$


since $i$ and $P_{q}$ are adjoints of each other by [5, (3.15)]. More importantly,

$$
U_{q, \phi}^{*}=\left(D_{q / 2}^{-q / 2}\right)^{*} T_{0, \phi}^{*}\left(D_{0}^{q / 2}\right)^{*}=D_{0}^{q / 2} T_{0, \bar{\phi}} D_{q / 2}^{-q / 2}=U_{q, \bar{\phi}}
$$

since

$$
\left(D_{s}^{t}\right)^{*}=\left(D_{s}^{t}\right)^{-1}=D_{s+t}^{-t}: \mathcal{F}_{q+2 t} \rightarrow \mathcal{F}_{q}
$$

by $[5,(3.6)]$ using $\llbracket \cdot, \cdot \rrbracket_{q}$. Further,

$$
\begin{aligned}
U_{q, z_{j}}^{*} z^{\alpha}= & \left(D_{0}^{q / 2} S_{0_{j}}^{*} D_{q / 2}^{-q / 2}\right) z^{\alpha}=\frac{\alpha_{j}}{N+|\alpha|} \frac{b_{|\alpha|-1}(q / 2)}{b_{|\alpha|-1}(0)} \frac{b_{|\alpha|}(0)}{b_{|\alpha|}(q / 2)} z^{\alpha+e_{j}}, \\
= & \begin{array}{ll}
\frac{\alpha_{j}}{N+q / 2+|\alpha|} z^{\alpha-e_{j}} & \text { if } q / 2>-(1+N), \\
\frac{(-(N+q / 2)+|\alpha|) \alpha_{j}}{|\alpha|^{2}} z^{\alpha-e_{j}} & \text { if } q / 2 \leq-(1+N),
\end{array}
\end{aligned}
$$

for $\alpha_{j}>0$. Of course $U_{q, z_{j}}^{*} z^{\alpha}=0$ if $\alpha_{j}=0$ and so $U_{q, z_{j}}^{*} 1=0$.

We state a few other properties of the $U_{q, \phi}$ obtained in [5, Theorem 4.5, Propositions 4.2 and 4.10] that we need. The first is just a repetition of (42), and the others follow from similar well-known results on Toeplitz operators on $\mathcal{F}_{0}$.

Theorem 10.1. (i) The operators $U_{q, \phi}$ and $T_{0, \phi}$ are unitarily equivalent via $D_{q / 2}^{-q / 2}$.

(ii) If $\phi \in L^{\infty}$, then $\left\|U_{q, \phi}\right\| \leq\|\phi\|_{L^{\infty}}$ using $\|\cdot\|_{q}$ on $\mathcal{F}_{q}$.

(iii) For $\phi \in C(\overline{\mathbb{B}}), U_{q, \phi}$ is compact if and only if $\phi$ restricts to 0 on $\partial \mathbb{B}$.

A straightforward computation shows that

$$
\begin{aligned}
U_{q, z_{j}} U_{q, z_{j}}^{*} z^{\alpha} & =\frac{\alpha_{j}}{N+|\alpha|} z^{\alpha}=S_{0_{j}} S_{0_{j}}^{*} z^{\alpha}, \\
U_{q, z_{j}}^{*} U_{q, z_{j}} z^{\alpha} & =\frac{1+\alpha_{j}}{1+N+|\alpha|} z^{\alpha}=S_{0_{j}}^{*} S_{0_{j}} z^{\alpha} .
\end{aligned}
$$

It follows that by picking those formulas and results in Secs. 6 and 9 for $S_{0}$, the shift operator on the unweighted Bergman space $\mathcal{F}_{0}$, we obtain for free many properties for $U_{q}=\left(U_{q, z_{1}}, \ldots, U_{q, z_{N}}\right)$ for any $q$.

Our main purpose in the section is to investigate the so-called Toeplitz algebras that we now define.

Definition 10.2. Let $q \in \mathbb{R}$. We denote by $\mathcal{T}_{q}$ the $C^{*}$-algebra generated by the shift operators $S_{q_{1}}, \ldots, S_{q_{N}}$ in $\mathcal{B}\left(\mathcal{F}_{q}\right)$. That is, $\mathcal{T}_{q}$ is the operator-norm closure of polynomials in $S_{q_{j}}$ and $S_{q_{j}}^{*}$.

Our study of $\mathcal{T}_{q}$ requires the study of two other similar algebras.

Definition 10.3. We denote by $\mathcal{U}_{q}$ the $C^{*}$-algebra generated by the Toeplitz operators $U_{q, z_{1}}, \ldots, U_{q, z_{N}}$. We denote by $\mathcal{V}_{q}$ the $C^{*}$-algebra of operators of the form $U_{q, \phi}+K$, where $\phi \in C(\overline{\mathbb{B}})$ and $K \in \mathcal{K}\left(\mathcal{F}_{q}\right)$.

Proposition 10.4. The collection $\mathcal{V}_{q}$ is in fact a $C^{*}$-algebra. 
Proof. Throughout, $\|\mid \cdot\|_{q}$ is used. Let $\phi, \psi \in C(\overline{\mathbb{B}})$. Then the semicommutator

$$
U_{q, \phi} U_{q, \psi}-U_{q, \phi \psi}=D_{0}^{q / 2}\left(T_{0, \phi} T_{0, \psi}-T_{0, \phi \psi}\right) D_{q / 2}^{-q / 2}=D_{0}^{q / 2} K_{1} D_{q / 2}^{-q / 2}=K_{2},
$$

where $K_{1} \in \mathcal{K}\left(\mathcal{F}_{0}\right)$ by the proof of [19, Theorem 1], and $K_{2} \in \mathcal{K}\left(\mathcal{F}_{q}\right)$. It follows that $\mathcal{V}_{q}$ is an algebra. It is clear that it is a $*$-algebra, that is, it is closed under taking adjoints. Aside, using the result on the semicommutator twice, the commutator $U_{q, \phi} U_{q, \psi}-U_{q, \psi} U_{q, \phi}$ is also compact.

Suppose $U_{q, \phi_{n}}+K_{n} \rightarrow X$ in norm. That is, $D_{0}^{q / 2}\left(T_{0, \phi_{n}}+L_{n}\right) D_{q / 2}^{-q / 2} \rightarrow X$ in norm, where $L_{n}=D_{q / 2}^{-q / 2} K_{n} D_{0}^{q / 2} \in \mathcal{K}\left(\mathcal{F}_{0}\right)$. So $T_{0, \phi_{n}}+L_{n} \rightarrow D_{q / 2}^{-q / 2} X D_{0}^{q / 2}=Y$. By [19, Theorem 1], $Y=T_{0, \phi_{0}}+K_{0}$ with $K_{0} \in \mathcal{K}\left(\mathcal{F}_{0}\right)$. Hence $X \in \mathcal{V}_{q}$ and $\mathcal{V}_{q}$ is norm-closed.

Since $\mathcal{V}_{q}$ lies in $\mathcal{B}\left(\mathcal{F}_{q}\right)$, we conclude that it is a $C^{*}$-algebra.

Proposition 10.5. The algebras $\mathcal{T}_{q}$ and $\mathcal{U}_{q}$ contain the identity operator.

Proof. The operator $S_{q}^{*} S_{q}$ lies in $\mathcal{T}_{q}$ and is invertible in $\mathcal{B}\left(\mathcal{F}_{q}\right)$ by $(28)$ and Theorem 3.5. By spectral permanence (see [22, Corollary I.5.7]), this inverse lies in the $C^{*}$ algebra generated by $S_{q}^{*} S_{q}$ which lies in $\mathcal{T}_{q}$. Thus $I \in \mathcal{T}_{q}$.

For $\mathcal{U}_{q}$, we use $U_{q}^{*} U_{q}=S_{0}^{*} S_{0}$ instead.

Corollary 10.6. The double centralizer (multiplier) algebra of $\mathcal{T}_{q}$ is itself.

Proof. See [14, Sec. 2] for the definition and basic properties of the double centralizer algebra of a $C^{*}$-algebra. By [14, Proposition 3.1], a $C^{*}$-algebra is a closed two-sided ideal in its double centralizer algebra.

Definition 10.7. A $C^{*}$-algebra $\mathcal{G} \subset \mathcal{B}(H)$ is called irreducible if there is no nontrivial (other than $\{0\}$ and $H$ ) subspace of $H$ which is invariant under all $A \in \mathcal{G}$. Equivalently, $\mathcal{G}$ is irreducible if there is no nontrivial orthogonal projection $P \in \mathcal{B}(H)$ commuting with all $A \in \mathcal{G}$.

Theorem 10.8. The algebras $\mathcal{T}_{q}$ and $\mathcal{U}_{q}$ are irreducible.

This result obviously is not new; see [29, Corollary 13] for a different proof.

Proof of Theorem 10.8. We follow [19, Lemma 1] which handles the cases $q=-1$ and $q=0$. Suppose $P$ is an orthogonal projection from $\mathcal{F}_{q}$ onto a closed subspace $W$ commuting with all the elements in $\mathcal{T}_{q}$. Let $g=P 1 \in W$. Then

$$
P S_{q_{j}}(1)=P\left(z_{j}\right)=S_{q_{j}} P(1)=S_{q_{j}}(g)=z_{j} g \quad(j=1, \ldots, N) .
$$

Repeating this with linear combinations of powers of $S_{q_{j}}$, we obtain that $P f=g f$ for any $f \in \mathcal{F}_{q}$, because linear combinations of powers of $\left\{z_{j}\right\}$ is dense in $\mathcal{F}_{q}$ by Definition 3.1. Thus $P=M_{g}$. But $\sigma(P)=\sigma\left(M_{g}\right) \subset\{0,1\}$. Since $g \in H(\mathbb{B})$, either 
$g \equiv 0$ or $g \equiv 1$. Equivalently, $P=0$ or $P=I$, proving that nontrivial orthogonal projections on $\mathcal{F}_{q}$ commuting with $\mathcal{T}_{q}$ do not exist.

For $\mathcal{U}_{q}$, we use Theorem 10.1(i). If $Q$ is an orthogonal projection from $\mathcal{F}_{q}$ onto a closed subspace $W$ commuting with everything in $\mathcal{U}_{q}$, then $Q U_{q, z_{j}}=U_{q, z_{j}} Q$ for each $j=1, \ldots, N$. Writing down the definition of $U_{q, z_{j}}$ and using (42), we obtain $P S_{q_{j}}=S_{q_{j}} P$ with $P=D_{q / 2}^{-q / 2} Q D_{0}^{q / 2}: \mathcal{F}_{0} \rightarrow D_{q / 2}^{-q / 2}(W) \subset \mathcal{F}_{0}$ a projection. If $g=Q 1$, let $h=P 1=D_{q / 2}^{-q / 2} g \in H(\mathbb{B})$. By the previous paragraph, $P=M_{h}$, and $P=I$ or $P=0$. Then also $Q=I$ or $Q=0$.

Proposition 10.9. The algebras $\mathcal{T}_{q}$ and $\mathcal{U}_{q}$ include the ideal $\mathcal{K}\left(\mathcal{F}_{q}\right)$.

Proof. The operators $I-S_{q} S_{q}^{*}$ and $I-U_{q} U_{q}^{*}=I-S_{0} S_{0}^{*}$ are nonzero, compact by Corollary 6.14 , and lie in $\mathcal{T}_{q}$ and $\mathcal{U}_{q}$, respectively. Then Theorem 10.8 in conjunction with [22, Corollary I.10.4] yields the desired result.

Proposition 10.10. The operator $U_{q, z_{j}}-S_{q_{j}}$ is compact and thus $\mathcal{T}_{q}=\mathcal{U}_{q}$.

Proof. By (40),

$\left(U_{q, z_{j}}-S_{q_{j}}\right) f= \begin{cases}\sum_{k=0}^{\infty} \frac{q / 2}{1+N+k} f_{k+1} & \text { if } q / 2>-(1+N), \\ \sum_{k=0}^{\infty} \frac{N^{2}+(1+N+k) q / 2}{(1+N+k)(1-(N+q / 2)+k)} f_{k+1} & \text { if } q / 2 \leq-(1+N) .\end{cases}$

By Theorem 3.5, $U_{q, z_{j}}-S_{q_{j}}$ is compact. Thus by Proposition $10.9, U_{q, z_{j}}$ lies in $\mathcal{T}_{q}$ and $S_{q_{j}}$ lies in $\mathcal{U}_{q}$.

Proposition 10.11. The algebras $\mathcal{V}_{q}$ and $\mathcal{U}_{q}$ are the same.

Proof. By Proposition 10.4, $\mathcal{U}_{q} \subset \mathcal{V}_{q}$. Conversely, let $U_{q, \phi} \in \mathcal{V}_{q}$, where $\phi \in C(\overline{\mathbb{B}})$. There is a sequence $\left\{p_{n}(z, \bar{z})\right\}$ of polynomials converging to $\phi$ uniformly on $\overline{\mathbb{B}}$ by the Stone-Weierstrass theorem. Since $\mathcal{U}_{q}$ is an algebra, $U_{q, p_{n}} \in \mathcal{U}_{q}$. By Theorem 10.1(ii), $U_{q, p_{n}} \rightarrow U_{q, \phi}$ in norm and thus $U_{q, \phi} \in \mathcal{U}_{q}$. By Proposition $10.9, \mathcal{K}\left(\mathcal{F}_{q}\right) \subset \mathcal{U}_{q}$. Therefore $\mathcal{V}_{q} \subset \mathcal{U}_{q}$ as well.

Therefore

$$
\mathcal{T}_{q}=\mathcal{V}_{q}=\mathcal{U}_{q} \quad(q \in \mathbb{R})
$$

Corollary 10.12. The commutator ideal of $\mathcal{T}_{q}$ is $\mathcal{K}\left(\mathcal{F}_{q}\right)$.

Proof. The commutator ideal of $\mathcal{T}_{q}$ is by definition the closed ideal generated by all operators of the form $A B-B A$ for $A, B \in \mathcal{T}_{q}$. By the proof of Proposition 10.4, the commutators and hence the commutator ideal, which is nonempty, lie in the 
ideal $\mathcal{K}\left(\mathcal{F}_{q}\right)$. But $\mathcal{K}\left(\mathcal{F}_{q}\right)$ is a simple $C^{*}$-algebra (see [22, Corollary I.10.3]), that is, it contains no proper nonzero closed two-sided ideal. The result follows.

Theorem 10.13. For every $q \in \mathbb{R}$,

$$
0 \longrightarrow \mathcal{K}\left(\mathcal{F}_{q}\right) \stackrel{i_{q}}{\longrightarrow} \mathcal{T}_{q} \stackrel{\gamma_{q}}{\longrightarrow} C(\partial \mathbb{B}) \longrightarrow 0
$$

is a short exact sequence of $C^{*}$-algebras. Here $i_{q}$ is the inclusion map and by definition $\gamma_{q}\left(U_{q, \phi}+K\right):=\left.\phi\right|_{\partial \mathbb{B}}$ using the identification $\mathcal{T}_{q}=\mathcal{V}_{q}$. In particular, $\gamma_{q}\left(S_{q_{j}}\right)=\gamma_{q}\left(U_{q, z_{j}}\right)=\left.z_{j}\right|_{\partial \mathbb{B}}$. Also, there is a continuous section $\eta_{q}: C(\partial \mathbb{B}) \rightarrow \mathcal{T}_{q}$.

Proof. Exact means that $i_{q}$ is one-to-one, $\gamma_{q}$ is onto, and $\operatorname{ker}\left(\gamma_{q}\right)=\operatorname{im}\left(i_{q}\right)$. The first is by definition, and the third is an immediate consequence of Theorem 10.1(iii). It is also clear that $i_{q}$ is a $*$-algebra homomorphism.

Given $\Phi, \Psi \in C(\partial \mathbb{B})$, extend them to $\overline{\mathbb{B}}$ using the Poisson integral and naming them $\phi, \psi$. Then $\gamma_{q}\left(U_{q, \phi}\right)=\Phi$ and $\gamma_{q}$ is onto. The boundary values of $\phi \psi$ and of the Poisson integral $\chi$ of $\Phi \Psi$ are both $\Phi \Psi$ making the difference $\phi \psi-\chi$ restrict to 0 on $\partial \mathbb{B}$. Then $U_{q, \chi}$ and $U_{q, \phi \psi}$ differ by an element of $\mathcal{K}\left(\mathcal{F}_{q}\right)$ by Theorem 10.1 (iii). This shows that $\gamma_{q}$ is an algebra homomorphism. It is also a $*$-homomorphism since the Poisson kernel is positive.

The action of $\gamma_{q}$ on $S_{q_{j}}$ is clear by Proposition 10.10 .

Thus $\mathcal{T}_{q} / \mathcal{K}\left(\mathcal{F}_{q}\right)$ and $C(\partial \mathbb{B})$ are isomorphic as $C^{*}$-algebras through the map $\iota_{q}\left(U_{q, \phi}+\mathcal{K}\left(\mathcal{F}_{q}\right)\right)=\left.\phi\right|_{\partial \mathbb{B}}$. There is the induced quotient map $\theta_{q}: \mathcal{T}_{q} \rightarrow \mathcal{T}_{q} / \mathcal{K}\left(\mathcal{F}_{q}\right)$ given by $\theta_{q}(A):=A+\mathcal{K}\left(\mathcal{F}_{q}\right)$.

Define $\eta_{q}: C(\partial \mathbb{B}) \rightarrow \mathcal{T}_{q}$ by $\eta_{q}(\Phi):=U_{q, \phi}$, which is continuous by Theorem 10.1(ii). Then $\theta_{q} \eta_{q}$ is a $*$-algebra homomorphism and $\iota_{q} \theta_{q} \eta_{q}=\gamma_{q} \eta_{q}=I$. This $\eta_{q}$ is the desired section.

The use of the Poisson integral in the proof is somewhat arbitrary. Instead, we could use the invariant Poisson integral, which also has a positive kernel.

Remark 10.14. Theorem 10.13 covers the special cases of the Drury-Arveson space $(q=-N,[9$, Theorem 5.7]), Hardy and Bergman spaces $(q=-1$ and $q=0$, [19, Theorem 1]), and the Dirichlet space $(q=-(1+N),[15$, Theorem 8] for $N=1$ ); after all, the definition of $\mathcal{T}_{q}$ is the same for all Hilbert spaces considered. Note that in the Hardy-space case, there is no need to extend continuous functions to all of $\overline{\mathbb{B}}$; most other sources work solely on $\partial \mathbb{B}$. In fact, the Hardy-space Toeplitz operators can be recovered in a limiting case as explained in [5, Remark 5.6]. Or, one can pass from the Hardy-space case $(q=-1)$ to the Bergman-space case $q=0$ as done in [19], and then pass to other $q$ as done above. The coverage mentioned holds for all the other results in this article as well unless specifically stated.

Short exact sequences have been used to investigate a variety of $C^{*}$-algebras of operators. See, for example, [30, Theorem 3.9] for those involving composition operators along with Toeplitz operators.

Corollary 10.15. The maximal ideal space of $\mathcal{T}_{q} / \mathcal{K}\left(\mathcal{F}_{q}\right)$ is $\partial \mathbb{B}$. 
Proof. The maximal ideal space of $C(K)$ is $K$.

Corollary 10.16. The Taylor joint essential spectrum of $S_{q}$ is $\partial \mathbb{B}$.

See [21, Definition 3.3] for the Taylor joint essential spectrum $\sigma_{T_{e}}$.

Proof of Corollary 10.16. We use [21, Corollary 3.10] whose essential normality condition on $S_{q}$ is provided by the second part of Proposition 9.5. Then $\sigma_{T_{e}}\left(S_{q}\right)$ is the maximal ideal space of $\mathcal{T}_{q} / \mathcal{K}\left(\mathcal{F}_{q}\right)$, which is identified in Corollary 10.15.

Proposition 10.17. We have $\left\|S_{q} S_{q}^{*}\right\|_{e}=\left\|S_{q}^{*} S_{q}\right\|_{e}=\|I\|=1$.

Proof. These are immediate by Corollary 6.14 .

Proposition 10.18. The identity representation of $\mathcal{T}_{q}$ is a boundary representation for the concrete operator space $\mathcal{S}_{q}=\operatorname{span}\left\{I, S_{q_{1}}, \ldots, S_{q_{N}}\right\}$ if and only if $q<-1$.

See $[9$, p. 205] for the definition of boundary representation.

Proof of Proposition 10.18. This has been done in [26] for $q \geq-(1+N)$; see the discussion on p. 220. The method of [9, Lemma 7.13] is applicable which takes care of the case $q=-N$. Various results in this section supply the conditions mentioned in [8, Theorem 2.1.1]. Then the proof boils down to determining those values of $q$ for which $\left\|S_{q}^{*} S_{q}\right\|>\left\|S_{q}^{*} S_{q}\right\|_{e}$ by [8, Remark, p. 284]. By (30) and Proposition 10.17, this is the case exactly for $q<-1$, even when $N=1$.

Corollary 10.19. If $q<-1$, then the $C^{*}$-envelope of $\mathcal{S}_{q}$ is the Toeplitz algebra $\mathcal{T}_{q}$.

See [28, Definition 2.5] for the definition of $C^{*}$-envelope.

Proof of Corollary 10.19. Clearly, $\mathcal{S}_{q}$ generates $\mathcal{T}_{q}$ as a $C^{*}$-algebra. By Proposition 10.18, the intersection of the kernels of all boundary representations of $\mathcal{S}_{q}$ is $\{0\}$ since the identity representation has that kernel. By [7, Theorem 2.2.3], the Shilov boundary of $\mathcal{S}_{q}$ is then $\{0\}$. By [28, Theorem 4.4, Proof], the $C^{*}$-envelope is the quotient of $\mathcal{T}_{q}$ by the Shilov boundary and is $\mathcal{T}_{q}$.

Definition 10.20. An operator $A \in \mathcal{B}(H)$ is called Fredholm if both $\operatorname{ker}(A)$ and $\operatorname{ker}\left(A^{*}\right)=\operatorname{im}(A)^{\perp}$ are finite-dimensional, in which case the Fredholm index of $A$ is defined as ind $(A)=\operatorname{dim} \operatorname{ker}(A)-\operatorname{dim} \operatorname{ker}\left(A^{*}\right)$. By the Atkinson theorem (see [38, Theorem 9.4.1]), equivalently, $A$ is Fredholm if $[A]$ is invertible in $\mathcal{Q}(H)$, and equivalently, $A$ is Fredholm if there exists a $B \in \mathcal{B}(H)$ such that $I-A B$ and $I-B A$ are compact.

When $N=1$, it is clear that every $S_{b}$ is Fredholm with $\operatorname{ind}\left(S_{b}\right)=-1$. But when $N>1$, no single shift operator $S_{b_{j}}$ is Fredholm, because $S_{b_{j}}^{*}$ annihilates the 
infinite-dimensional subspace of $\mathcal{F}_{b}$ consisting of those $f$ whose Taylor series (12) have $f_{\alpha}=0$ whenever $\alpha_{j}>0$.

On the other hand, with Notation $6.1, S_{b} S_{b}^{*}$ is Fredholm with $\operatorname{ind}\left(S_{b} S_{b}^{*}\right)=0$ since it is self-adjoint and its kernel is one-dimensional, and $S_{b}^{*} S_{b}$ is Fredholm with $\operatorname{ind}\left(S_{b}^{*} S_{b}\right)=0$ since it is invertible. The latter fact is equivalent to the Fredholmness and the invertibility of the tuple $S_{b}$ by [21, Corollary 3.9]; see [21, Definition 3.1] for the definition.

Proposition 10.21. The operator $I-S_{b} S_{b}^{*}$ is Fredholm if and only if all except finitely many of the $b_{k}$ are different. The operator $I-S_{b}^{*} S_{b}$ is Fredholm if and only if $(1+k) b_{k+1} \neq(N+k) b_{k}$ for all except finitely many $k$, which reduces to the condition in the previous sentence for $N=1$. The operator $I-S_{q} S_{q}^{*}$ is Fredholm if and only if $q \neq-N$. The operator $I-S_{q}^{*} S_{q}$ is Fredholm if and only if $q \neq-1$. In each case that an operator is Fredholm, its index is 0.

Proof. It is enough to have a look at the explicit forms of the operators on $f \in \mathcal{F}_{b}$ or $f \in \mathcal{F}_{q}$ in Sec. 6 .

Corollary 10.22. For $\phi \in C(\overline{\mathbb{B}})$ and $K \in \mathcal{K}\left(\mathcal{F}_{q}\right), U_{q, \phi}+K$ is Fredholm if and only if $\phi$ does not vanish on $\partial \mathbb{B}$.

Proof. By spectral permanence, $U_{q, \phi}+K$ is a Fredholm operator if and only if $\left[U_{q, \phi}+K\right]=\left[U_{q, \phi}\right]$ is invertible in $\mathcal{T}_{q} / \mathcal{K}\left(\mathcal{F}_{q}\right)$. By Theorem 10.13, this quotient is isomorphic to $C(\partial \mathbb{B})$. Thus $U_{q, \phi}+K$ is Fredholm if and only if $\left.\phi\right|_{\partial \mathbb{B}}$ is invertible, that is, if and only if this restriction is never 0 .

Remark 10.23. If $A$ and $B$ are unitarily equivalent and $A$ is Fredholm, then $B$ is also Fredholm with $\operatorname{ind}(A)=\operatorname{ind}(B)$. Thus the Fredholm properties of $U_{q, \phi}$ can in general be surmised from those of $T_{0, \phi}$ by Theorem 10.1(i).

Corollary 10.24. If $\phi \in C(\overline{\mathbb{B}})$, then $\sigma_{e}\left(U_{q, \phi}\right)=\sigma_{e}\left(T_{q, \phi}\right)=\phi(\partial \mathbb{B})$.

Proof. It suffices to do the proof for $U_{q, \phi}$ only. If $\lambda \notin \phi(\partial \mathbb{B})$, then $U_{q, \phi}-\lambda I$ is invertible in $C(\partial \mathbb{B})$. Equivalently, $U_{q, \phi}-\lambda I+\mathcal{K}\left(\mathcal{F}_{q}\right)=\left[U_{q, \phi}-\lambda I\right]$ is invertible in $\mathcal{T}_{q} / \mathcal{K}(H)$ by Theorem 10.13. By spectral permanence, equivalently, $\left[U_{q, \phi}-\lambda I\right]$ is invertible in $\mathcal{Q}(H)$. Hence $\lambda \notin \sigma_{e}\left(U_{q, \phi}\right)$.

Conversely, let $\lambda \in \phi(\partial \mathbb{B})$. Without loss of generality, $\phi(1,0, \ldots, 0)=\lambda=0$. We need to show that $U_{q, \phi}$ is not Fredholm. If it were Fredholm, then $T_{0, \phi}$ would also be Fredholm by Remark 10.23. Then the construction in the proof of $[45$, Theorem $6.5]$ with $z$ replaced by $z_{1}$ would yield a contradiction.

Proposition 10.25. Suppose $\phi \in C(\overline{\mathbb{B}})$ and $U_{q, \phi}$ is Fredholm. If $N>1$, then $\operatorname{ind}\left(U_{q, \phi}\right)=0$. If $N=1$, then $\operatorname{ind}\left(U_{q, \phi}\right)=-n$, where $n$ is the winding number of the closed curve $\phi(\partial \mathbb{D})$ about 0 . 
Proof. By Corollary 10.22, $\phi$ does not vanish on $\partial \mathbb{B}$. For $N>1, \mathbb{B}$ is strongly pseudoconvex and has simply connected boundary. Then [43, Theorem 1.4] shows that $\operatorname{ind}\left(T_{0, \phi}\right)=0$. By Remark 10.23, also $\operatorname{ind}\left(U_{q, \phi}\right)=0$. For $N=1$, by [43, Theorem 1.3], $\operatorname{ind}\left(T_{0, \phi}\right)=-n$. Then by Remark 10.23, also $\operatorname{ind}\left(U_{q, \phi}\right)=-n$.

Corollary 10.26. The short exact sequence in Theorem 10.13 is not split exact if $N=1$.

Proof. Split exact means that there is a $*$-algebra homomorphism $\kappa_{q}: C(\partial \mathbb{D}) \rightarrow$ $\mathcal{T}_{q}$ such that $\gamma_{q} \kappa_{q}=I$. Suppose that is the case. Let $\Phi \in C(\partial \mathbb{D})$ be given by $\Phi(\zeta)=\zeta=\left.z\right|_{\partial \mathbb{D}}$ and let $N_{q}=\kappa_{q}(\Phi) \in \mathcal{T}_{q}$. By assumption, $\gamma_{q}\left(N_{q}\right)=\gamma_{q}\left(U_{q, z}\right)=\zeta$. On the other hand, since $\kappa_{q}(\bar{\Phi})=N_{q}^{*}$ and $\bar{\Phi}$ commutes with $\Phi, N_{q}$ is normal. Then $N_{q}-U_{q, z}=K_{q} \in \operatorname{ker}\left(\gamma_{q}\right)=\operatorname{im}\left(i_{q}\right)=\mathcal{K}\left(\mathcal{F}_{q}\right)$. Also $U_{q, z}=D_{0}^{q / 2} T_{0, z} D_{q / 2}^{-q / 2}$ is Fredholm since $T_{0, z}=S_{0}$ is. But then $N_{q}$ is also Fredholm and these imply the impossibility $0=\operatorname{ind}\left(N_{q}\right)=\operatorname{ind}\left(U_{q, z}\right)=-1$ by Proposition 10.25.

We finish this section with a different kind of result.

Theorem 10.27. The algebra $\mathcal{T}_{q}$ is dense in $\mathcal{B}\left(\mathcal{F}_{q}\right)$ in the strong operator topology.

Proof. Let $A \in \mathcal{B}\left(\mathcal{F}_{q}\right)$. Consider Corollary 4.8 with $q+2 t=0$ and $s=q / 2$; then $t=-q / 2$ and $u=0$. Then as in the discussion at the beginning of this section, $D_{q / 2}^{-q / 2} A D_{0}^{q / 2} \in \mathcal{B}\left(\mathcal{F}_{0}\right)$. By $\left[24\right.$, Corollary 3], the collection $\left\{T_{0, \phi}: \phi \in C(\overline{\mathbb{B}})\right\}$ is dense in $\mathcal{B}\left(\mathcal{F}_{0}\right)$ in the same topology. So there is a sequence $\left\{\phi_{n}\right\}$ with $\phi_{n} \in C(\overline{\mathbb{B}})$ such that $\left\|T_{0, \phi_{n}} g-D_{q / 2}^{-q / 2} A D_{0}^{q / 2} g\right\|_{0} \rightarrow 0$ as $n \rightarrow \infty$ for all $g \in \mathcal{F}_{0}$. Put $f=D_{0}^{q / 2} g$; then $f \in \mathcal{F}_{q}$ and is arbitrary. By (39), we have $\left\|D_{q / 2}^{-q / 2} U_{q, \phi_{n}} f-D_{q / 2}^{-q / 2} A f\right\|_{0} \rightarrow 0$ for all $f \in \mathcal{F}_{q}$. This is the same as $\left\|U_{q, \phi_{n}} f-A f\right\|_{q} \rightarrow 0$ as $n \rightarrow \infty$ for all $f \in \mathcal{F}_{q}$. We are done by $\left\{U_{q, \phi}: \phi \in C(\overline{\mathbb{B}})\right\} \subset \mathcal{V}_{q}=\mathcal{T}_{q}$.

\section{Ext Classes}

This section is devoted to proving that all the short exact sequences indexed by $q \in \mathbb{R}$ in Theorem 10.13 are in the same Ext class in two different ways. We notice that the maps that realize these are the radial differential operators that have been so useful already. In the process, we compute the Busby maps by interpreting the short exact sequences as extensions of $C(\partial \mathbb{B})$. We also identify the $K$-theory groups $K_{0}\left(\mathcal{T}_{q}\right)$ and $K_{1}\left(\mathcal{T}_{q}\right)$; see [44] for their definitions and properties.

The bases of Proposition 3.4, when written for the $\mathcal{F}_{q}$ using (18), take the form

$$
O_{\alpha}^{q}(z)= \begin{cases}\frac{\sqrt{(1+N+q)|\alpha|}}{\sqrt{\alpha !}} z^{\alpha} & \text { if } q>-(1+N), \\ \frac{|\alpha| !}{\sqrt{(1-(N+q))_{|\alpha|} \alpha !}} z^{\alpha} & \text { if } q \leq-(1+N),\end{cases}
$$


and are orthonormal with respect to $\|\cdot\|_{q}$. Given $q_{1} \neq q_{2}$, define $Q: \mathcal{F}_{q_{1}} \rightarrow \mathcal{F}_{q_{2}}$ by $Q\left(O_{\alpha}^{q_{1}}\right):=O_{\alpha}^{q_{2}}$. This is a unitary operator with respect to $[\cdot, \cdot]_{q}$ by definition, and

$$
Q z^{\alpha}= \begin{cases}\sqrt{\frac{\left(1+N+q_{2}\right)|\alpha|}{\left(1+N+q_{1}\right)|\alpha|}} z^{\alpha} & \text { if } q>-(1+N), \\ \sqrt{\frac{\left(1-\left(N+q_{1}\right)\right)_{|\alpha|}}{\left(1-\left(N+q_{2}\right)\right)_{|\alpha|}}} z^{\alpha} & \text { if } q \leq-(1+N) .\end{cases}
$$

The coefficient of $z^{\alpha}$ depends only on $|\alpha|$; calling it $g_{|\alpha|}$, we see that if $f \in \mathcal{F}_{q_{1}}$, then $Q f=\sum_{k=0}^{\infty} g_{k} f_{k}$, where $g_{k} \sim k^{\left(q_{2}-q_{1}\right) / 2}$. Thus $Q$ is a radial differential operator of order $\left(q_{2}-q_{1}\right) / 2$ much like those in Sec. 4 .

However, as in Sec. 10, we continue to use $\|\mid \cdot\|_{q}$ and $\llbracket \cdot, \cdot \rrbracket_{q}$, and the correct radial differential operator to use with them is not $Q$ but $D_{q_{1} / 2}^{\left(q_{2}-q_{1}\right) / 2}: \mathcal{F}_{q_{1}} \rightarrow \mathcal{F}_{q_{2}}$ which is bounded and onto by Corollary 4.8 . By (42), it is also unitary with respect to $\llbracket \cdot, \cdot \rrbracket_{q}$, and

$$
\left(D_{q_{1} / 2}^{\left(q_{2}-q_{1}\right) / 2}\right)^{*}=\left(D_{q_{1} / 2}^{\left(q_{2}-q_{1}\right) / 2}\right)^{-1}=D_{q_{2} / 2}^{\left(q_{1}-q_{2}\right) / 2}: \mathcal{F}_{q_{2}} \rightarrow \mathcal{F}_{q_{1}} .
$$

It can be checked that $D_{q_{1} / 2}^{\left(q_{2}-q_{1}\right) / 2}$ takes an orthonormal basis to another with respect to $\|\cdot \mid \cdot\|_{q}$.

Proposition 11.1. For every $q_{1}, q_{2} \in \mathbb{R}$, there is a unital $*$-algebra isomorphism $\Omega$ between the $C^{*}$-algebras $\mathcal{K}\left(\mathcal{F}_{q_{1}}\right)$ and $\mathcal{K}\left(\mathcal{F}_{q_{2}}\right)$, between $\mathcal{B}\left(\mathcal{F}_{q_{1}}\right)$ and $\mathcal{B}\left(\mathcal{F}_{q_{2}}\right)$, and between $\mathcal{T}_{q_{1}}$ and $\mathcal{T}_{q_{2}}$, which induces a *-algebra isomorphism between $\mathcal{Q}\left(\mathcal{F}_{q_{1}}\right)$ and $\mathcal{Q}\left(\mathcal{F}_{q_{2}}\right)$.

Proof. If $A$ is an operator in one of the $C^{*}$-algebras with parameter $q_{1}$, define $\Omega(A):=D_{q_{1} / 2}^{\left(q_{2}-q_{1}\right) / 2} A D_{q_{2} / 2}^{\left(q_{1}-q_{2}\right) / 2}$. Then $\Omega(A)$ lies in the corresponding $C^{*}$-algebra with parameter $q_{2}$. This $\Omega$ is invertible with $\Omega^{-1}(B)=D_{q_{2} / 2}^{\left(q_{1}-q_{2}\right) / 2} B D_{q_{1} / 2}^{\left(q_{2}-q_{1}\right) / 2}$ by (43), which also shows that $\Omega$ is an algebra isomorphism. The fact that $\Omega$ respects the *-operation follows from the positivity of the $D_{s}^{t}$ stated in Proposition 4.4. Clearly, $\Omega(I)=I$.

The map $\Omega$ is often written $\operatorname{Ad}_{D_{q_{1} / 2}^{\left(q_{2}-q_{1}\right) / 2}}$ in the literature.

Corollary 11.2. For every $q \in \mathbb{R}$, two groups arising in $K$-theory are $K_{0}\left(\mathcal{T}_{q}\right)=\mathbb{Z}$ and $K_{1}\left(\mathcal{T}_{q}\right)=\{0\}$

Proof. Cao [16, p. 178] gives these groups for $q=-1$; Remark 10.14 is relevant here. But these groups are invariant under homotopy equivalence between unital $C^{*}$-algebras, hence under *-algebra isomorphisms; see [38, Propositions 3.2.6 and 8.2.2]. Proposition 11.1 finishes the proof.

For only integer $q \geq-1$, this result is in [11, Proposition 3.1]. 
A short exact sequence as in Theorem 10.13, or the triple $\left(i_{q}, \mathcal{T}_{q}, \gamma_{q}\right)$, is often called an extension of $C(\partial \mathbb{B})$ by $\mathcal{K}\left(\mathcal{F}_{q}\right)$. There are two notions of equivalence between extensions in $[13,15.4]$ that we use. The first is the following.

Definition 11.3. We say two extensions of a $C^{*}$-algebra $\mathcal{G}$ by $\mathcal{H}_{1}$ and $\mathcal{H}_{2}$ with middle terms $\mathcal{E}_{1}$ and $\mathcal{E}_{2}$ are weakly isomorphic if there are $*$-algebra isomorphisms $\xi_{1}: \mathcal{G} \rightarrow \mathcal{G}, \xi_{2}: \mathcal{E}_{1} \rightarrow \mathcal{E}_{2}, \xi_{3}: \mathcal{H}_{1} \rightarrow \mathcal{H}_{2}$ such that these maps and the maps of the extensions commute.

Theorem 11.4. For every $q_{1}, q_{2} \in \mathbb{R}$, extensions of $C(\partial \mathbb{B})$ by $\mathcal{K}\left(\mathcal{F}_{q_{1}}\right)$ and $\mathcal{K}\left(\mathcal{F}_{q_{2}}\right)$ with middle terms $\mathcal{T}_{q_{1}}$ and $\mathcal{T}_{q_{2}}$ are weakly isomorphic.

Proof. Define $\xi_{1}=I$ and $\xi_{2}=\xi_{3}=\Omega$ of Proposition 11.1. Then we have the following diagram:

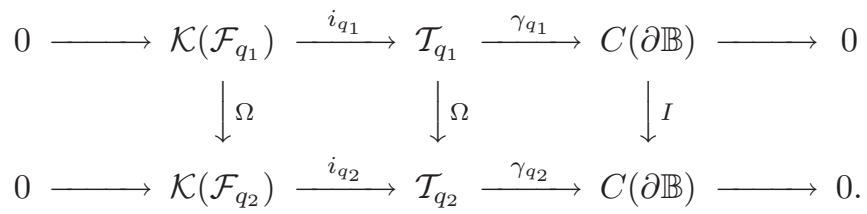

But by (39) and (20),

$$
\begin{aligned}
U_{q_{2}, \phi} & =D_{0}^{q_{2} / 2} T_{0, \phi} D_{q_{2} / 2}^{-q_{2} / 2}=D_{q_{1} / 2}^{\left(q_{2}-q_{1}\right) / 2} D_{0}^{q_{1} / 2} T_{0, \phi} D_{q_{1} / 2}^{-q_{1} / 2} D_{q_{2} / 2}^{\left(q_{1}-q_{2}\right) / 2} \\
& =D_{q_{1} / 2}^{\left(q_{2}-q_{1}\right) / 2} U_{q_{1}, \phi} D_{q_{2} / 2}^{\left(q_{1}-q_{2}\right) / 2}=\Omega\left(U_{q_{1}, \phi}\right) .
\end{aligned}
$$

The maps on the left square commute trivially by Proposition 11.1. For the commutativity on the right square, let $U_{q_{1}, \phi}+K_{1} \in \mathcal{T}_{q_{1}}$ with $K_{1} \in \mathcal{K}\left(\mathcal{F}_{q_{1}}\right)$. Then $\gamma_{q_{2}}\left(\Omega\left(U_{q_{1}, \phi}+K_{1}\right)\right)=\gamma_{q_{2}}\left(U_{q_{2}, \phi}+K_{2}\right)=\left.\phi\right|_{\partial \mathbb{B}}$, where $K_{2} \in \mathcal{K}\left(\mathcal{F}_{q_{2}}\right)$. But also $I\left(\gamma_{q_{1}}\left(U_{q_{1}, \phi}+K_{1}\right)\right)=\left.\phi\right|_{\partial \mathbb{B}}$.

There is also the standard short exact sequence

$$
0 \longrightarrow \mathcal{K}\left(\mathcal{F}_{q}\right) \stackrel{i_{q}}{\longrightarrow} \mathcal{B}\left(\mathcal{F}_{q}\right) \stackrel{\pi_{q}}{\longrightarrow} \mathcal{Q}\left(\mathcal{F}_{q}\right) \longrightarrow 0
$$

of $C^{*}$-algebras, where the inclusion map $i_{q}$ and the quotient map $\pi_{q}$ are $*$-algebra homomorphisms. If $A \in \mathcal{B}\left(\mathcal{F}_{q}\right)$, then $\pi_{q}(A)=[A]$ and $\pi_{q}(A)^{*}=\pi_{q}\left(A^{*}\right)$. We also use a version of the standard short exact sequence in which operators act between different Hilbert spaces, which is

$$
0 \longrightarrow \mathcal{K}\left(\mathcal{F}_{q_{1}} \rightarrow \mathcal{F}_{q_{2}}\right) \stackrel{i_{q_{1} q_{2}}}{\longrightarrow} \mathcal{B}\left(\mathcal{F}_{q_{1}} \rightarrow \mathcal{F}_{q_{2}}\right) \stackrel{\pi_{q_{1} q_{2}}}{\longrightarrow} \mathcal{Q}\left(\mathcal{F}_{q_{1}} \rightarrow \mathcal{F}_{q_{2}}\right) \longrightarrow 0
$$

Associated to each extension of a $C^{*}$-algebra $\mathcal{G}$ by $\mathcal{K}\left(\mathcal{F}_{q}\right)$ with middle term $\mathcal{E}$, there are $*$-algebra homomorphisms $\rho_{q}$ and $\tau_{q}$ as shown making the diagram 
commute, and $\tau_{q}$ is called the Busby map (see [14]):

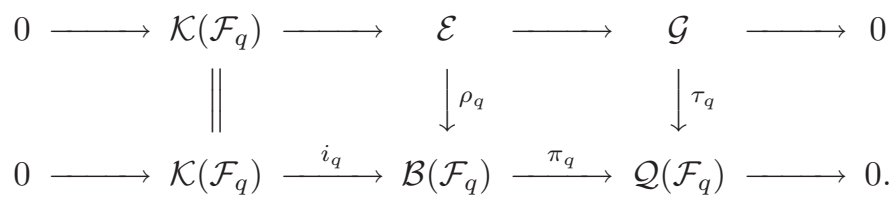

The Busby map is an invariant of the extension, that is, each determines the other; see $[13,15.3]$.

Given $\Phi \in C(\partial \mathbb{B})$, let $\phi$ be its Poisson integral. Working out the details of $\left[33\right.$, Example 3.1.2] with $\mathcal{G}=C(\partial \mathbb{B}), \mathcal{E}=\mathcal{T}_{q}$, and $i_{q}$ and $\gamma_{q}$ as in Theorem 10.13, we compute that $\rho_{q}=i_{q}$ and that the Busby map of the extension in Theorem 10.13 has the precise form

$$
\tau_{q}(\Phi)=\pi_{q}\left(U_{q, \phi}\right)=\left[U_{q, \phi}\right]
$$

the equivalence class of the generalized Toeplitz operator $U_{q, \phi}$ in $\mathcal{Q}\left(\mathcal{F}_{q}\right)$. This $\tau_{q}$ is one-to-one, because if $\tau_{q}(\Phi)=[0]=\mathcal{K}\left(\mathcal{F}_{q}\right)$, then $U_{q, \phi}$ is compact which happens precisely when $\Phi=0$ by Theorem 10.1(iii). This has a consequence. An ideal of a $C^{*}$-algebra is called essential if it intersects every other nonzero ideal nontrivially. By [44, Proposition 3.2.15], we conclude the following.

Corollary 11.5. The ideal $\mathcal{K}\left(\mathcal{F}_{q}\right)$ is essential in $\mathcal{T}_{q}$.

In this connection, let us recall that the maximal unital $C^{*}$-algebra in which $\mathcal{K}\left(\mathcal{F}_{q}\right)$ is essential is its double centralizer (multiplier) algebra $\mathcal{B}\left(\mathcal{F}_{q}\right)$; see $[13,12.1]$.

The second equivalence notion in $[13,15.4]$ that we use is the following.

Definition 11.6. We say two extensions of a $C^{*}$-algebra $\mathcal{G}$ by $\mathcal{K}\left(\mathcal{F}_{q_{1}}\right)$ and $\mathcal{K}\left(\mathcal{F}_{q_{2}}\right)$ with Busby maps $\tau_{q_{1}}$ and $\tau_{q_{2}}$ are strongly unitarily equivalent if there is a unitary operator $V: \mathcal{F}_{q_{1}} \rightarrow \mathcal{F}_{q_{2}}$ such that for all $A \in \mathcal{G}$, we have

$$
\tau_{q_{2}}(A)=\pi_{q_{1} q_{2}}(V) \tau_{q_{1}}(A) \pi_{q_{1} q_{2}}(V)^{*} \quad(A \in \mathcal{G})
$$

in $\mathcal{Q}\left(\mathcal{F}_{q_{2}}\right)$. We write $\operatorname{Ext}(\mathcal{G})$ for the equivalence classes of extensions of $\mathcal{G}$ under this notion.

Theorem 11.7. For every $q_{1}, q_{2} \in \mathbb{R}$, extensions of $C(\partial \mathbb{B})$ by $\mathcal{K}\left(\mathcal{F}_{q_{1}}\right)$ and $\mathcal{K}\left(\mathcal{F}_{q_{2}}\right)$ are strongly unitarily equivalent. In other words, all these extensions represent the same class in $\operatorname{Ext}(C(\partial \mathbb{B}))$.

Proof. Let $\Phi \in C(\partial \mathbb{B})$ and $\phi$ be its Poisson integral as usual. By (44), we have $U_{q_{2}, \phi}=D_{q_{1} / 2}^{\left(q_{2}-q_{1}\right) / 2} U_{q_{1}, \phi} D_{q_{2} / 2}^{\left(q_{1}-q_{2}\right) / 2}$. Define $V: \mathcal{F}_{1} \rightarrow \mathcal{F}_{2}$ as $V=D_{q_{1} / 2}^{\left(q_{2}-q_{1}\right) / 2}$. It is unitary by (43). Apply $\pi_{q_{2}}$ to the equality between the Toeplitz operators, use the 
fact that it is a homomorphism, and recall (45). Then

$$
\begin{aligned}
\tau_{q_{2}}(\Phi) & =\pi_{q_{2}}\left(U_{q_{2}, \phi}\right)=\pi_{q_{2}}\left(V U_{q_{1}, \phi} V^{*}\right) \\
& =\pi_{q_{1} q_{2}}(V) \pi_{q_{1}}\left(U_{q_{1}, \phi}\right) \pi_{q_{2} q_{1}}\left(V^{*}\right)=\pi_{q_{1} q_{2}}(V) \tau_{q_{1}}(\Phi) \pi_{q_{1} q_{2}}(V)^{*} .
\end{aligned}
$$

We have achieved more than we have been aiming. The equality (46) holds not only at the equivalence class level, but also at the operator level with the $V$ chosen. Thus a radial differential operator plays a key role once again.

A similar theorem involving $C^{*}$-algebras generated by Toeplitz and composition operators together, but only with $q=0$ and $q=-1$, is in [30, Theorem 4.1].

\section{Acknowledgments}

The author dedicates this paper to the memory of Walter Rudin and of Mary Ellen Rudin who wrote their only joint paper [39] after their retirement from teaching. The author thanks Baruch Solel of the Technion for asking the right question, Aurelian Gheondea of Bilkent University for several useful discussions, Joseph A. Ball of Virginia Tech for updating him on the literature, Thomas L. Kriete and Barbara D. MacCluer of the University of Virginia for their wonderful lectures, and an anonymous referee for pointing out a significant gap in a proof. The author is deeply indebted to and generously thanks Semra Öztürk Kaptanoğlu of Middle East Technical University for the creative discussions that made the mathematical content of this paper considerably richer.

\section{References}

[1] J. Agler, Hypercontractions and subnormality, J. Operator Theory 13 (1985) 203-217.

[2] J. Agler and J. E. McCarthy, Pick Interpolation and Hilbert Function Spaces, Graduate Studies in Mathematics, Vol. 44 (American Mathematical Society, Providence, RI, 2002).

[3] D. Alpay and H. T. Kaptanoğlu, Integral formulas for a sub-Hardy Hilbert space on the ball with complete Nevanlinna-Pick reproducing kernel, C. R. Acad. Sci. Paris Sér. I Math. 333 (2001) 285-290.

[4] Gleason's problem and homogeneous interpolation in Hardy and Dirichlettype spaces of the ball, J. Math. Anal. Appl. 276 (2002) 654-672.

[5] Toeplitz operators on Arveson and Dirichlet spaces, Integral Equations Operator Theory 58 (2007) 1-33.

[6] J. Arazy and G. Zhang, Homogeneous multiplication operators on bounded symmetric domains, J. Funct. Anal. 202 (2003) 44-66.

[7] W. Arveson, Subalgebras of $C^{*}$-algebras, Acta Math. 123 (1969) 141-224.

[8] Subalgebras of $C^{*}$-algebras II, Acta Math. 128 (1972) 271-308.

[9] , Subalgebras of $C^{*}$-algebras III: Multivariable operator theory, Acta Math. 181 (1998) 159-228.

[10] A. Athavale, Model theory on the unit ball of $\mathbb{C}^{m}$, J. Operator Theory 27 (1992) $347-358$. 
[11] Equations Operator Theory 68 (2010) 255-262.

[12] F. Beatrous and J. Burbea, On multipliers for Hardy-Sobolev spaces, Proc. Amer. Math. Soc. 136 (2008) 2125-2133.

[13] B. Blackadar, K-Theory for Operator Algebras, 2nd edn., Mathematical Sciences Research Institute Publications, Vol. 5 (Cambridge University Press, Cambridge, 1998).

[14] R. C. Busby, Double centralizers and extensions of $C^{*}$-algebras, Trans. Amer. Math. Soc. 132 (1968) 79-99.

[15] G. Cao, Fredholm properties of Toeplitz operators on Dirichlet spaces, Pacific J. Math. 188 (1999) 209-223.

[16] - Toeplitz algebras on strongly pseudoconvex domains, Nagoya Math. J. 185 (2007) 171-186.

[17] X. Chen and K. Guo, Analytic Hilbert Modules, Research Notes in Mathematics (Chapman \& Hall/CRC, Boca Raton, FL, 2003).

[18] M. Chō and W. Żelazko, On geometric spectral radius of commuting $n$-tuples of operators, Hokkaido Math. J. 21 (1992) 251-258.

[19] L. A. Coburn, Singular integral operators and Toeplitz operators on odd spheres, Indiana Univ. Math. J. 23 (1973) 433-439.

[20] J. B. Conway, A Course in Operator Theory, Graduate Studies in Mathematics, Vol. 21 (American Mathematical Society, Providence, RI, 2000).

[21] R. E. Curto, Fredholm and invertible $n$-tuples of operators: The deformation problem, Trans. Amer. Math. Soc. 266 (1981) 129-159.

[22] K. R. Davidson, $C^{*}$-Algebras by Example, Fields Institute Monographs, Vol. 6 (American Mathematical Society, Providence, RI, 1996).

[23] S. W. Drury, A generalization of von Neumann inequality to the complex ball, Proc. Amer. Math. Soc. 68 (1978) 300-304.

[24] M. Engliš, Density of algebras generated by Toeplitz operators on Bergman spaces, Ark. Mat. 30 (1992) 227-243.

[25] J. Gleason, S. Richter and C. Sundberg, On the index of invariant subspaces in spaces of functions of several complex variables, J. Reine Angew. Math. 587 (2005) 49-76.

[26] K. Guo, J. Hu and X. Xu, Toeplitz algebras, subnormal tuples and rigidity on reproducing $\mathbb{C}\left[z_{1}, \ldots, z_{d}\right]$-modules, J. Funct. Anal. 210 (2004) 214-247.

[27] K. Guo and K. Wang, Essentially normal Hilbert modules and K-homology, Math. Ann. 340 (2008) 907-934.

[28] M. Hamana, Injective envelopes of operator systems, Publ. Res. Inst. Math. Sci. 15 (1979) $773-785$.

[29] N. P. Jewell and A. R. Lubin, Commuting weighted shifts and analytic function theory in several variables, J. Operator Theory 1 (1979) 207-223.

[30] M. T. Jury, $C^{*}$-algebras generated by groups of composition operators, Indiana Univ. Math. J. 56 (2007) 3171-3192.

[31] H. T. Kaptanoğlu, Bergman projections on Besov spaces on balls, Illinois J. Math. 49 (2005) 385-403.

[32] _ Reproducing kernels and radial differential operators for holomorphic and harmonic Besov spaces on unit balls: A unified view, Comput. Methods Funct. Theory 10 (2010) 483-500.

[33] G. J. Murphy, $C^{*}$-Algebras and Operator Theory (Academic Press, San Diego, CA, 1990).

[34] V. Müller and A. Sołtysiak, Spectral radius formula for commuting Hilbert space operators, Studia Math. 103 (1992) 329-333. 
[35] V. Müller and F.-H. Vasilescu, Standard models for some commuting multioperators, Proc. Amer. Math. Soc. 117 (1993) 979-989.

[36] J. M. Ortega and J. Fàbrega, Pointwise multipliers and decomposition theorems in analytic Besov spaces, Math. Z. 235 (2000) 53-81.

[37] S. Pott, Standard models under polynomial positivity conditions, J. Operator Theory 41 (1999) 365-389.

[38] M. Rørdam, F. Larsen and N. J. Laustsen, An Introduction to K-Theory for $C^{*}$ Algebras, London Mathematical Society Student Texts, Vol. 49 (Cambridge University Press, Cambridge, 2000).

[39] M. E. Rudin and W. Rudin, Continuous functions that are locally constant on dense sets, J. Funct. Anal. 133 (1995) 129-137.

[40] W. Rudin, Principles of Mathematical Analysis, 3rd edn., International Series in Pure and Applied Mathematics (McGraw-Hill, New York, 1976).

[41] D. Sarason, Generalized interpolation in $H^{\infty}$, Trans. Amer. Math. Soc. 127 (1967) 179-203.

[42] A. L. Shields, Weighted shift operators and analytic function theory, in Topics in Operator Theory, ed. C. Pearcy, Mathematical Surveys, Vol. 13 (American Mathematical Society, Providence, RI, 1974), pp. 49-128.

[43] U. Venugopalkrishna, Fredholm operators associated with strongly pseudoconvex domains in $\mathbb{C}^{n}$, J. Funct. Anal. 9 (1972) 349-373.

[44] N. E. Wegge-Olsen, K-Theory and $C^{*}$-Algebras: A Friendly Approach (Oxford University Press, New York, 1993).

[45] X. Zeng, Toeplitz operators on Bergman spaces, Houston J. Math. 18 (1992) 387-407. 